\title{
KAC-MOODY LIE ALGEBRAS, SPECTRAL SEQUENCES, AND THE WITT FORMULA
}

\author{
SEOK-JIN KANG
}

\begin{abstract}
In this work, we develop a homological theory for the graded Lie algebras, which gives new information on the structure of the Lorentzian KacMoody Lie algebras. The technique of the Hochschild-Serre spectral sequences offers a uniform method of studying the higher level root multiplicities and the principally specialized affine characters of Lorentzian Kac-Moody Lie algebras.
\end{abstract}

\section{INTRODUCTION}

In the past 20 years, the theory of Kac-Moody Lie algebras has developed rapidly and with great success. Surprising connections to areas such as combinatorics, modular forms, and mathematical physics have shown Kac-Moody Lie algebras to be of uncommon interest. The discovery of the Macdonald identities gave rise to an intensive study of the class of affine Kac-Moody Lie algebras and their representations [Mcd]. The structure of such Lie algebras and their connections with other branches of mathematics and mathematical physics have been well-established and are being extensively investigated.

The next natural step after the affine case is that of the hyperbolic Kac-Moody Lie algebras. One of the most ambitious goals of current research activity in infinite dimensional Lie algebras may be to construct geometric realizations of the hyperbolic Kac-Moody Lie algebras. Once that is accomplished, we will have a much deeper understanding of the structure of Kac-Moody Lie algebras and their connections with number theory. Unfortunately, many basic questions regarding the hyperbolic case are still unresolved. For example, the behavior of the root multiplicities is not well-understood. Feingold-Frenkel [F-F] and KacMoody-Wakimoto [K-M-W] made some progress in this area. They computed the level 2 root multiplicities for the hyperbolic Kac-Moody Lie algebras $H A_{1}^{(1)}$ and $H E_{8}^{(1)}$. Other important works on the hyperbolic Kac-Moody Lie algebras include [Fe2, Fr, LM, and M2]. Recently, V. Kac has informed the author that he also discovered a level 3 root multiplicity formula for $H A_{1}^{(1)}$ (unpublished).

In this work, we develop a homological theory for the graded Lie algebras. Combining with the representation theory of affine Kac-Moody Lie algebras, we obtain new information on the structure of the Lorentzian Kac-Moody Lie algebras; i.e., Kac-Moody Lie algebras whose Cartan matrix has a Lorentzian

Received by the editors May 21, 1990 and, in revised form, April 26, 1991.

1991 Mathematics Subject Classification. Primary 17B65, 17B67, 17 B10.

This work was supported by NSF grant DMS 8806371 . 
signature. The technique of the Hochschild-Serre spectral sequences offers a uniform method of studying the higher level root multiplicities and the principally specialized affine characters of Lorentzian Kac-Moody Lie algebras.

More precisely, we start with the observation that any symmetrizable KacMoody Lie algebra can be realized as the minimal graded Lie algebra $L=$ $\bigoplus_{n \in Z} L_{n}$ with the local part $V \oplus L_{0} \oplus V^{*}$, where $L_{0}$ is a "smaller" Kac-Moody Lie algebra, $V$ is an integrable irreducible highest weight module over $L_{0}$, and $V^{*}$ is the contragredient of $V$ in a suitable sense [Kang]. So $L=G / I$, where $G=\bigoplus_{n \in Z} G_{n}$ is the maximal graded Lie algebra with the local part $V \oplus L_{0} \oplus V^{*}$, and $I=\bigoplus_{n \in Z} I_{n}$ is the maximal graded ideal of $G$ intersecting the local part trivially. In particular, when $L_{0}$ is an affine Kac-Moody Lie algebra and $V$ is the basic representation of $L_{0}$, we obtain a series of Lorentzian Kac-Moody Lie algebras (e.g., [F-F, K-M-W, Kang]). The idea is to study the structure of each homogeneous space $L_{n}$ as a module over the affine Kac-Moody Lie algebra $L_{0}$. We note that $G_{ \pm}=\bigoplus_{n \geq 1} G_{ \pm n}$ is the free Lie algebra generated by $G_{1}=V^{*}$ (respectively, $G_{-1}=V$ ). Thus we divide our study into two parts: the study of the free Lie algebra and the study of the maximal graded ideal.

For the free Lie algebra, by a direct generalization of the proof of the classical Witt formula given in [Se], we obtain a character formula for the free Lie algebra, which we also call the Witt formula. The main ingredients of the proof are the Poincaré-Birkhoff-Witt theorem and the Möbius inversion. For the maximal graded ideal, as $\mathrm{O}$. Mathieu pointed out (in private communication), one can use the exact sequences in [Kac2] to understand the structure of $I$. The main result of this work is the following reduction theorem:

Let $I$ be the graded ideal of the free Lie algebra $G$ generated by the subspace $I_{m}$ of $G_{m}$ for $m \geq 2$. Let $I^{(j)}=\sum_{n \geq j \geq m} I_{n}$. Since $I_{m}$ generates $I, I^{(j)}$ is also a graded ideal of $G$ generated by the subspace $I_{j}$. Consider the quotient Lie algebra $L^{(j)}=G / I^{(j)}$. Then we have

$$
I_{j+1} \cong\left(V \otimes I_{j}\right) / H_{3}\left(L^{(j)}\right)_{j+1} .
$$

Thus we reduce the problem to computing $H_{3}\left(L^{(j)}\right)$. When $j$ is the first nontrivial index, we can compute the homology modules using the Kostant formula [Ko, G-L, Liu]. For the higher levels, we invoke the technique of HochschildSerre spectral sequences and the five term exact sequences. Combining with the representation theory of affine Kac-Moody Lie algebras, we determine some of the boundary homomorphisms, and deduce new structural information on the maximal graded ideal. Applying this to certain Lorentzian Kac-Moody Lie algebras, we compute the principally specialized affine characters for certain higher levels. Furthermore, we compute the root multiplicities of the hyperbolic KacMoody Lie algebras $H A_{1}^{(1)}$ and $H A_{2}^{(2)}$ up to level 3. All the formulas obtained here are new. Comparing with Kac's result, we obtain a combinatorial identity. As far as we know, the formulas for levels higher than 3 are first computed here.

\section{Preliminaries}

An $n \times n$ matrix $A=\left(a_{i j}\right)$ is called a generalized Cartan matrix if it satisfies the following conditions: (i) $a_{i i}=2$ for $i=1, \ldots, n$, (ii) $a_{i j}$ are nonpositive integers for $i \neq j$, (iii) $a_{i j}=0$ implies $a_{j i}=0 . A$ is called symmetrizable if $D A$ is symmetric for some diagonal matrix $D=\operatorname{diag}\left(q_{1}, \ldots, q_{n}\right)$ with $q_{i}>0$, 
$q_{i} \in \mathbf{Q}$. A realization of an $n \times n$ matrix $A$ of rank $l$ is a triple $\left(\mathfrak{h}, \Pi, \Pi^{\vee}\right)$, where $\mathfrak{h}$ is a $(2 n-l)$-dimensional complex vector space, $\Pi=\left\{\alpha_{1}, \ldots, \alpha_{n}\right\}$ and $\Pi^{\vee}=\left\{\alpha_{1}^{\vee}, \ldots, \alpha_{n}^{\vee}\right\}$ are linearly independent indexed subsets of $\mathfrak{h}^{*}$ and $\mathfrak{h}$, respectively, satisfying $\alpha_{j}\left(\alpha_{i}^{\vee}\right)=a_{i j}$ for $i, j=1, \ldots, n$. The Kac-Moody Lie algebra $\mathfrak{g}(A)$ associated with a generalized Cartan matrix $A$ is the Lie algebra generated by the elements $e_{i}, f_{i}(i=1, \ldots, n)$ and $\mathfrak{h}$ with the following defining relations:

$$
\begin{aligned}
{\left[h, h^{\prime}\right] } & =0 \text { for } h, h^{\prime} \in \mathfrak{h}, \\
{\left[e_{i}, f_{j}\right] } & =\delta_{i j} \alpha_{i}^{\vee} \text { for } i, j=1, \ldots, n, \\
{\left[h, e_{j}\right] } & =\alpha_{j}(h) e_{j}, \quad\left[h, f_{j}\right]=-\alpha_{j}(h) f_{j} \quad \text { for } j=1, \ldots, n, \\
\left(\text { ade }_{i}\right)^{1-a_{i j}}\left(e_{j}\right) & =0 \text { for } i, j=1, \ldots, n \text { with } i \neq j, \\
\left(\text { adf }_{i}\right)^{1-a_{i j}}\left(f_{j}\right) & =0 \text { for } i, j=1, \ldots, n \text { with } i \neq j .
\end{aligned}
$$

The Kac-Moody Lie algebra $\mathfrak{g}(A)$ has the root space decomposition

$$
\mathfrak{g}(A)=\bigoplus_{\alpha \in \mathfrak{h}^{*}} \mathfrak{g}_{\alpha},
$$

where

$$
\mathfrak{g}_{\alpha}=\{x \in \mathfrak{g}(A) \mid[h, x]=\alpha(h) x \text { for all } h \in \mathfrak{h}\} .
$$

An element $\alpha \in \mathfrak{h}^{*}$ is called a root if $\mathfrak{g}_{\alpha} \neq 0$. The space $\mathfrak{g}_{\alpha}$ is called the $\alpha$-root space and $\operatorname{dim} \mathfrak{g}_{\alpha}$ is called the multiplicity of $\alpha$. Denote by $\mathfrak{n}^{+}$(respectively, $\mathfrak{n}^{-}$) the subalgebra of $\mathfrak{g}(A)$ generated by the elements $e_{i}$ (respectively, $f_{i}$ ) for $i=1, \ldots, n$. Then $\mathfrak{g}(A)$ has the triangular decomposition $\mathfrak{g}(A)=\mathfrak{n}^{-} \oplus \mathfrak{h} \oplus \mathfrak{n}^{+}$.

A $\mathfrak{g}(A)$-module $V$ is called a highest weight module with highest weight $\Lambda \in$ $\mathfrak{h}^{*}$ if there exists a nonzero vector $v \in V$ such that (i) $\mathfrak{n}^{+} \cdot v=0$, (ii) $h \cdot v=$ $\Lambda(h) v$ for all $h \in \mathfrak{h}$, (iii) $U(\mathfrak{g}(A)) \cdot v=V$, where $U(\mathfrak{g}(A))$ denotes the universal enveloping algebra of $\mathfrak{g}(A)$. A highest weight module $V$ with highest weight $\Lambda$ has the weight space decomposition

$$
V=\bigoplus_{\lambda \in \mathfrak{h}^{*}} V_{\lambda}
$$

where

$$
V_{\lambda}=\{v \in V \mid h \cdot v=\lambda(h) v \text { for all } h \in \mathfrak{h}\} .
$$

It is easy to see that $\operatorname{dim} V_{\lambda}<\infty$. We define the formal character of $V$ to be

$$
\operatorname{ch} V=\sum_{\lambda \in h^{*}}\left(\operatorname{dim} V_{\lambda}\right) e(\lambda) \text {, }
$$

where $e(\lambda)$ are the elements of the group algebra $\mathbf{C}\left[\mathfrak{h}^{*}\right]$ with the multiplication

$$
e(\lambda) e(\mu)=e(\lambda+\mu) \text { for } \lambda, \mu \in \mathfrak{h}^{*} .
$$

For a weight $\lambda=\Lambda-\sum_{i} k_{i} \alpha_{i}$ of $V$, we define $\operatorname{deg}(\lambda)=\sum_{i} k_{i}$. Then setting

$$
V_{j}=\bigoplus_{\lambda: \operatorname{deg}(\lambda)=j} V_{\lambda}
$$

defines the principal gradation of $V$ :

$$
V=\bigoplus_{j \geq 0} V_{j} .
$$


We define the principally specialized character of $V$ to be

$$
\operatorname{ch}_{q}=\sum_{j \geq 0}\left(\operatorname{dim} V_{j}\right) q^{j} .
$$

For each $\Lambda \in \mathfrak{h}^{*}$, there exists a unique irreducible highest weight module $V(\Lambda)$ with highest weight $\Lambda$. If $\Lambda$ is dominant integral, $V(\Lambda)$ is integrable; i.e., all the $e_{i}$ and $f_{i}(i=1, \ldots, n)$ are locally nilpotent on $V(\Lambda)$.

An indecomposable generalized Cartan matrix $A$ is said to be of finite type if all its principal minors are positive, of affine type if all its proper principal minors are positive and $\operatorname{det} A=0$, and of indefinite type if $A$ is of neither finite nor affine type. $A$ is of hyperbolic type if it is of indefinite type and all its proper principal submatrices are of finite or affine type.

Let $A=\left(a_{i j}\right)_{i, j=0,1, \ldots, l}$ be a generalized Cartan matrix of affine type and let $\tilde{A}=\left(\tilde{a}_{i j}\right)_{i, j=-1,0,1, \ldots, l}$ be the generalized Cartan matrix defined by

$$
\begin{aligned}
\tilde{a}_{-1,-1} & =2, \quad \tilde{a}_{-1,0}=\tilde{a}_{0,-1}=-1, \\
\tilde{a}_{-1, i} & =\tilde{a}_{i,-1}=0, \quad \text { for } i=1, \ldots, l, \\
\tilde{a}_{i, j} & =a_{i, j}, \quad \text { for } i, j=0,1, \ldots, l .
\end{aligned}
$$

Then $\tilde{A}$ has a Lorentzian signature. The Kac-Moody Lie algebras whose Cartan matrix has a Lorentzian signature are said to be of Lorentzian type. Note that the matrix $\tilde{A}$ is of hyperbolic type for small values of $n$. In this work, we study the structure of Kac-Moody Lie algebras $\mathfrak{g}(\widetilde{A})$ of Lorentzian type, where $\tilde{A}$ has the form (1.2).

Let $V=V\left(\Lambda_{0}\right)$ be the basic representation of the affine Kac-Moody Lie algebra $\mathfrak{g}(A)$ and let $V^{*}$ be the contragredient of $V$ in the sense that $V^{*}$ is the space of all linear functionals on $V$ which vanish on all but finitely many weight spaces of $V$. Hence $V^{*}$ is the irreducible lowest representation of $\mathfrak{g}(A)$ with lowest weight $-\Lambda_{0}$. Let $\langle$,$\rangle denote the canonical bilinear pairing$ between $V^{*}$ and $V$. For $x \in \mathfrak{g}(A), v \in V, v^{*} \in V^{*}$, we simply write $\left\langle v^{*} x v\right\rangle$ for

$$
\left\langle v^{*} \cdot x, v\right\rangle=-\left\langle x \cdot v^{*}, v\right\rangle=\left\langle v^{*}, x \cdot v\right\rangle .
$$

Choose a pair of dual bases $\left\{x_{i} \mid i \in J\right\}$ and $\left\{y_{i} \mid i \in J\right\}$ of $\mathfrak{g}(A)$ with respect to the standard invariant symmetric bilinear form defined on $\mathfrak{g}(A)$ [Kac2, Chapter 2]. Define a bilinear map $\phi: V^{*} \times V \rightarrow \mathfrak{g}(A)$ by

$$
\phi\left(v^{*}, v\right)=-\sum_{i \in J}\left\langle v^{*} x_{i} v\right\rangle y_{i} .
$$

It is clear that the map $\phi$ is well-defined. The space $V \oplus \mathfrak{g}(A) \oplus V^{*}$ has a local Lie algebra structure with the Lie bracket defined as follows.

(i) The Lie bracket in $\mathfrak{g}(A)$ is the obvious one.

(ii) The Lie bracket between $\mathfrak{g}(A)$ and $V$ (respectively, $V^{*}$ ) is given by the $\mathfrak{g}(A)$-module action:

$[x, v]=x \cdot v, \quad\left[x, v^{*}\right]=x \cdot v^{*}, \quad$ for $v \in V, v^{*} \in V^{*}$, and $x \in \mathfrak{g}(A)$.

(iii) The bracket between $V$ and $V^{*}$ is given by the map $\phi: V^{*} \times V \rightarrow \mathfrak{g}(A)$ :

$$
\left[v^{*}, v\right]=\phi\left(v^{*}, v\right) \text { for } v^{*} \in V^{*} \text { and } v \in V \text {. }
$$


Thus there exist the maximal graded Lie algebra $G=\bigoplus_{n \in Z} G_{n}$ and the minimal graded Lie algebra $L=\bigoplus_{n \in Z} L_{n}$ with the local part $V \oplus \mathfrak{g}(A) \oplus V^{*}$ [Kacl]. Note that $G_{0}=L_{0}=\mathfrak{g}(A)$. For $n \geq 1$, the homogeneous subspace $G_{n}$ (respectively, $G_{-n}$ ) is spanned by all the brackets of $n$ vectors from $V^{*}$ (respectively, $V$ ), and the grading on $L$ is induced by that of $G$. We define a graded ideal $I$ as follows. For $n \geq 2$, let

$$
I_{ \pm n}=\left\{x \in G_{ \pm n} \mid\left(\operatorname{ad} G_{\mp 1}\right)^{n-1} x=0\right\},
$$

and define $I=\bigoplus_{n \in Z} I_{n}$. Set $I_{ \pm}=\bigoplus_{n \geq 1} I_{ \pm n}$. Then the subspaces $I$ and $I_{ \pm}$ are all graded ideals of $G$, and $I$ is the maximal graded ideal of $G$ intersecting the local part trivially [B-K-M, F-F, Kacl, Kang]. Hence $L=G / I$. We write $G_{ \pm}=\bigoplus_{n \geq 1} G_{ \pm n}$ and $L_{ \pm}=\bigoplus_{n>1} L_{ \pm n}$. Then $G_{+}$(respectively, $G_{-}$) is the free Lie algebra generated by $V^{*}$ (respectively, $V$ ).

Using the Gabber-Kac Theorem [G-K], we can prove that $L$ is isomorphic to the Kac-Moody Lie algebra $\mathfrak{g}(\tilde{A})$ [F-F, K-M-W, Kang]. In particular, the ideal $I_{+}$(respectively, $\left.I_{-}\right)$is generated by the elements $\left(\mathrm{ad} v_{0}^{*}\right)^{2}\left(e_{i}\right)$ (respectively, $\left.\left(\operatorname{ad} v_{0}\right)^{2}\left(f_{i}\right)\right)$ for $i=0,1, \ldots, l$, where $v_{0}$ and $v_{0}^{*}$ are highest and lowest weight vectors of $V$ and $V^{*}$, respectively. Thus the ideal $I_{+}$(respectively, $I_{-}$) is generated by the space $I_{2}$ (respectively, $I_{-2}$ ). We denote by $\alpha_{-1}, \alpha_{0}, \ldots, \alpha_{l}$ the simple roots of $\mathfrak{g}(A)$. Thus $V$ is the irreducible highest weight module over $\mathfrak{g}(A)$ with highest weight $-\alpha_{-1}$ and $V^{*}$ is the irreducible lowest weight module over $\mathfrak{g}(A)$ with lowest weight $\alpha_{-1}$. We will study the structure of the homogeneous subspaces $L_{n}=G_{n} / I_{n}$ as modules over the affine Kac-Moody Lie algebra $\mathfrak{g}(A)$.

\section{THE WitT FORMULA}

Let $G=\bigoplus_{n>1} G_{n}$ be the free Lie algebra with a finite set of (free) generators $\left\{x_{1}, \ldots, x_{r}\right\}$. Then we have the following well-known Witt formula [J2]:

$$
\operatorname{dim} G_{n}=\frac{1}{n} \sum_{d \mid n} \mu(d) r^{n / d},
$$

where $\mu$ denotes the classical Möbius function. A nice proof is given in [Se]. The main ingredients of the proof are the Poincaré-Birkhoff-Witt theorem and Möbius inversion.

More generally, let $X=\left\{x_{i} \mid i=1,2,3, \ldots\right\}$ be a totally ordered set (possibly countably infinite) and let $R$ be an (additive) partially ordered abelian semigroup with a countable basis such that each element $\alpha$ of $R$ can be expressed as a sum of elements of $R$ which are less than or equal to $\alpha$ in only finitely many ways. Let $G$ be the free Lie algebra on the set $X$. We make $G$ an $R$-graded Lie algebra as follows. Define $\operatorname{deg}\left(x_{i}\right)=\mu_{i}$ for $\mu_{i} \in R$ such that $\mu_{i} \leq \mu_{j}$ for $i<j$, and

$$
\operatorname{deg}\left(\left[\left[\cdots\left[x_{i_{1}}, x_{i_{r}}\right] \cdots\right] x_{i_{r}}\right]\right)=\mu_{i_{1}}+\cdots+\mu_{i_{r}} .
$$

Let $G_{\lambda}=\{x \in G \mid \operatorname{deg}(x)=\lambda\}$ for $\lambda \in R$. Then $G$ has the decomposition $G=\bigoplus_{\lambda \in R} G_{\lambda}$, and $\left[G_{\lambda}, G_{\mu}\right] \subset G_{\lambda+\mu}$ for $\lambda, \mu \in R$. If $G_{\lambda} \neq 0$, we call $\lambda$ a root of $G$ and $G_{\lambda}$ the $\lambda$-root space of $G$. When all the root spaces are finite dimensional, we define the formal character of $G$ to be

$$
\operatorname{ch} G=\sum_{\lambda \in R} \operatorname{dim} G_{\lambda} e(\lambda),
$$


where $e(\lambda)$ are the elements of the semigroup algebra $\mathbf{C}[R]$ with the multiplication $e(\lambda) e(\mu)=e(\lambda+\mu)$. Similarly, we can define the roots, root spaces, and formal character for the universal enveloping algebra $U(G)$ of $G$. Then by generalizing the proof of the Witt formula given in [Se], we obtain the following generalization:

Theorem 2.1. Let $S=\left\{\tau_{i} \mid i=1,2,3, \ldots\right\}$ be the set of distinct degrees of the elements of $X$. Then the vector space $V$ spanned by the elements of $X$ has the following decomposition: $V=\bigoplus_{i=1}^{\infty} V_{\tau_{i}}$. For $\tau \in R$, set

$$
T(\tau)=\left\{(n)=\left(n_{1}, n_{2}, n_{3}, \ldots\right) \mid n_{i} \in \mathbf{Z}_{\geq 0}, \sum n_{i} \tau_{i}=\tau\right\},
$$

and define

$$
B(\tau)=\sum_{(n) \in T(\tau)} \frac{\left(\left(\sum n_{i}\right)-1\right) !}{\prod\left(n_{i} !\right)} \prod\left(\operatorname{dim} V_{\tau_{i}}\right)^{n_{i}}
$$

Then

$$
\operatorname{dim} G_{\lambda}=\sum_{\tau \mid \lambda} \mu\left(\frac{\lambda}{\tau}\right) \frac{\tau}{\lambda} B(\tau),
$$

where $\tau \mid \lambda$ if $\lambda=k \tau$ for some positive integer $k$, in which case $\lambda / \tau=k$ and $\tau / \lambda=1 / k$.

Proof. Let $\gamma_{1}, \gamma_{2}, \gamma_{3}, \ldots$ be an enumeration of elements of $R$ and let $m_{s}=$ $\operatorname{dim} G_{\gamma_{s}}$. For each $s=1,2,3, \ldots$, let $\left\{e_{\gamma_{s}, i_{s}} \mid i_{s}=1, \ldots, m_{s}\right\}$ be a basis of $G_{\gamma_{s}}$. Then by the Poincaré-Birkhoff-Witt theorem the vectors of the form

$$
e_{\gamma_{1}, 1}^{n_{1,1}} \cdots e_{\gamma_{1}, m_{1}}^{n_{1}, m_{1}} e_{\gamma_{2}, 1}^{n_{2,1}} \cdots e_{\gamma_{2}, m_{2}}^{n_{2, m_{2}}} \cdots e_{\gamma_{t}, 1}^{n_{t, 1}} \cdots e_{\gamma_{t}, m_{t}}^{n_{t, m_{t}}}
$$

are a basis of $U(G)$. Thus $\operatorname{dim} U(G)_{\lambda}$ is the cardinality of the family $\left\{n_{i, j}\right\}$ such that

$$
\left(n_{1,1}+\cdots+n_{1, m_{1}}\right) \gamma_{1}+\left(n_{2,1}+\cdots+n_{2, m_{2}}\right) \gamma_{2}+\cdots=\lambda .
$$

But this is the coefficient of $e(\lambda)$ in the following formal expression:

$$
\prod_{s=1}^{\infty}\left(1+e\left(\gamma_{s}\right)+e\left(2 \gamma_{s}\right)+e\left(3 \gamma_{s}\right)+\cdots\right)^{m_{s}}
$$

Therefore

$$
\begin{aligned}
\operatorname{ch} U(G) & =\sum_{\lambda \in R}\left(\operatorname{dim} U(G)_{\lambda}\right) e(\lambda) \\
& =\prod_{s=1}^{\infty}\left(1+e\left(\gamma_{s}\right)+e\left(2 \gamma_{s}\right)+e\left(3 \gamma_{s}\right)+\cdots\right)^{m_{s}} \\
& =\prod_{s=1}^{\infty} \frac{1}{\left(1-e\left(\gamma_{s}\right)\right)^{m_{s}}}=\prod_{\lambda \in R}(1-e(\lambda))^{-\operatorname{dim} G_{\lambda}} .
\end{aligned}
$$

On the other hand, let $V$ be the vector space spanned by the set $X$. Then $U(G)$ is the tensor algebra $T(V)=\mathbf{C} \oplus V \oplus(V \otimes V) \oplus \cdots$ on $V$. Thus we have $\operatorname{ch} U(G)=1+\operatorname{ch} V+(\operatorname{ch} V)^{2}+\cdots=\frac{1}{1-\operatorname{ch} V}=\frac{1}{1-\sum_{i=1}^{\infty}\left(\operatorname{dim} V_{\tau_{i}}\right) e\left(\tau_{i}\right)}$. 
Therefore we obtain

$$
\operatorname{ch} U(G)=\frac{1}{1-\sum_{i=1}^{\infty}\left(\operatorname{dim} V_{\tau_{i}}\right) e\left(\tau_{i}\right)}=\prod_{\lambda \in R}(1-e(\lambda))^{-\operatorname{dim} G_{\lambda}}
$$

Using the formal power series $\log (1-t)=-\sum_{m=1}^{\infty} t^{m} / m$, we get from the left-hand side

$$
\begin{aligned}
\log \left(\frac{1}{1-\operatorname{ch} V}\right) & =-\log (1-\operatorname{ch} V)=\sum_{m=1}^{\infty} \frac{(\operatorname{ch} V)^{m}}{m} \\
& =\sum_{m=1}^{\infty} \frac{1}{m}\left(\sum_{i=1}^{\infty}\left(\operatorname{dim} V_{\tau_{i}}\right) e\left(\tau_{i}\right)\right)^{m} \\
& =\sum_{m=1}^{\infty} \frac{1}{m} \sum_{(n)=\left(n_{i}\right)} \frac{\left(\sum n_{i}\right) !}{\prod\left(n_{i} !\right)} \prod\left(\operatorname{dim} V_{\tau_{i}}\right)^{n_{i}} e\left(\sum n_{i} \tau_{i}\right) \\
& =\sum_{\tau \in R} \sum_{(n) \in T(\tau)} \frac{\left(\left(\sum n_{i}\right)-1\right) !}{\prod\left(n_{i} !\right)} \prod\left(\operatorname{dim} V_{\tau_{i}}\right)^{n_{i}} e(\tau) \\
& =\sum_{\tau \in R} B(\tau) e(\tau) .
\end{aligned}
$$

From the right-hand side, we have

$$
\begin{aligned}
\log \left(\prod_{\lambda \in R}(1-e(\lambda))^{-\operatorname{dim} G_{\lambda}}\right)=\sum_{\lambda \in R}\left(\operatorname{dim} G_{\lambda}\right) \log \left(\frac{1}{1-e(\lambda)}\right) \\
=\sum_{\lambda \in R}\left(\operatorname{dim} G_{\lambda}\right) \sum_{k=1}^{\infty} \frac{e(k \lambda)}{k}=\sum_{\lambda \in R}\left(\sum_{k=1}^{\infty} \frac{1}{k}\left(\operatorname{dim} G_{\lambda}\right) e(k \lambda)\right) .
\end{aligned}
$$

Therefore

$$
B(\tau)=\sum_{\lambda: \tau=k \lambda} \frac{1}{k} \operatorname{dim} G_{\lambda}=\sum_{\lambda \mid \tau}\left(\frac{\lambda}{\tau}\right) \operatorname{dim} G_{\lambda} .
$$

Hence by Möbius inversion

$$
\operatorname{dim} G_{\lambda}=\sum_{\tau \mid \lambda} \mu\left(\frac{\lambda}{\tau}\right) \frac{\tau}{\lambda} B(\tau)
$$

Remark 2.2. We call the function $B(\tau)$ the Witt partition function on $V$. The formula (2.3) will also be called the Witt formula.

Let $V=V(\Lambda)$ be an integrable irreducible highest weight module over a symmetrizable Kac-Moody Lie algebra $\mathfrak{g}$, and let $G=\bigoplus_{n \geq 1} G_{n}$ be the free Lie algebra generated by $V$. Then $G$ is also an integrable module over $\mathfrak{g}$, and $G$ has the root space decomposition induced by the weight space decomposition of $V$. Let $R=\mathfrak{h}^{*}$ with the usual partial ordering [Kac2]. Let $S=\left\{\tau_{i} \mid i=\right.$ $1,2,3, \ldots\}$ be an enumeration of all the weights of $V$. Then by the Witt formula,

$$
\operatorname{dim} G_{\lambda}=\sum_{\tau \mid \lambda} \mu\left(\frac{\lambda}{\tau}\right) \frac{\tau}{\lambda} B(\tau)
$$

for $\lambda \in \mathfrak{h}^{*}$. 
Consider the principal gradation on $V=\bigoplus_{m \geq 0} V_{m}$. Then each homogeneous space $G_{n}$ is also principally graded induced by the principal gradation of $V$ : $G_{n}=\bigoplus_{m \geq 0} G_{(n, m)}$. We give a new gradation to $V$ by setting

$$
\widehat{V}_{m+1}=V_{m} \text { for } m \geq 0 \text {. }
$$

Thus we have $V=\bigoplus_{j \geq 1} \widehat{V}_{j}$. Then this induces a new gradation on $G_{n}$ :

$$
G_{n}=\bigoplus_{j \geq n} \widehat{G}_{(n, j)},
$$

where $\widehat{G}_{(n, j)}$ are given by

$$
\widehat{G}_{(n, n+m)}=G_{(n, m)} \text { for } m \geq 0 .
$$

Let $R=\{(r, s) \in \mathbf{Z} \times \mathbf{Z} \mid r \geq 1, s \geq 1\}$ with the lexicographic ordering. Let $S=\left\{(1, j) \mid j \in \mathbf{Z}_{\geq 1}\right\}$ and for a pair of positive integers $r, s$, let

$$
T(r, s)=\left\{(n)=\left(n_{1}, n_{2}, n_{3}, \ldots\right) \mid n_{j} \in \mathbf{Z}_{\geq 0}, \sum n_{j}=r, \sum j n_{j}=s\right\} .
$$

Note that the set $T(r, s)$ corresponds to the set of partitions of $s$ into $r$ parts. Then by the Witt formula, we obtain

$$
\begin{aligned}
\operatorname{dim} G_{(n, m)} & =\operatorname{dim} \widehat{G}_{(n, n+m)} \\
& =\sum_{(r, s) \mid(n, n+m)} \mu\left(\frac{(n, n+m)}{(r, s)}\right) \frac{(r, s)}{(n, n+m)} \widehat{B}(r, s),
\end{aligned}
$$

where $\widehat{B}(r, s)$ is defined by

$$
\begin{aligned}
\widehat{B}(r, s) & =\sum_{(n) \in T(r, s)} \frac{\left(\left(\sum n_{j}\right)-1\right) !}{\prod\left(n_{j} !\right)} \prod\left(\operatorname{dim} \widehat{V}_{j}\right)^{n_{j}}, \\
& =\sum_{(n) \in T(r, s)} \frac{\left(\left(\sum n_{j}\right)-1\right) !}{\prod\left(n_{j} !\right)} \prod\left(\operatorname{dim} V_{j-1}\right)^{n_{j}} .
\end{aligned}
$$

Hence the principally specialized character of $G_{n}$ is given by

$$
\begin{aligned}
\operatorname{ch}_{q} G_{n} & =\sum_{m \geq 0}\left(\operatorname{dim} G_{(n, m)}\right) q^{m} \\
& =\sum_{m \geq 0}\left(\sum_{(r, s) \mid(n, n+m)} \mu\left(\frac{(n, n+m)}{(r, s)}\right) \frac{(r, s)}{(n, n+m)} \widehat{B}(r, s)\right) q^{m} .
\end{aligned}
$$

\section{HoCHSCHILD-SERRE SPECTRAL SEQUENCES}

Let $G$ be a Lie algebra and $V$ a module over $G$. We define the space $C_{q}(G, V)$ of $q$-dimensional chains of the Lie algebra $G$ with coefficients in $V$ to be $\Lambda^{q}(G) \otimes V$. The differential $d_{q}: C_{q}(G, V) \rightarrow C_{q-1}(G, V)$ is defined by the formula

$$
\begin{aligned}
d_{q}\left(g_{1}\right. & \left.\wedge \cdots \wedge g_{q} \otimes v\right) \\
= & \sum_{1 \leq s<t \leq q}(-1)^{s+t-1}\left(\left[g_{s}, g_{t}\right] \wedge g_{1} \wedge \cdots \wedge \hat{g}_{s} \wedge \cdots \wedge \hat{g}_{t} \wedge \cdots \wedge g_{q}\right) \otimes v \\
& +\sum_{1 \leq s \leq q}(-1)^{s}\left(g_{1} \wedge \cdots \wedge \hat{g}_{s} \wedge \cdots \wedge g_{q}\right) \otimes g_{s} \cdot v
\end{aligned}
$$


where $v \in V, g_{1}, \ldots, g_{q} \in G$. For $q<0$, we define $C_{q}(G, V)=0$ and $d_{q}=0$. Then we have $d_{q} \circ d_{q+1}=0$. The homology of the complex $(C, d)=$ $\left\{C_{q}(G, V), d_{q}\right\}$ is called the homology of the Lie algebra $G$ with coefficients in $V$, and is denoted by $H_{q}(G, V)$. When $V=\mathrm{C}$, we write $H_{q}(G)$ for $H_{q}(G, \mathrm{C})$.

We shall be interested in the cases where $G, V$, and $C_{q}(G, V)$ are completely reducible modules in category $\mathscr{O}$ over a Kac-Moody. Lie algebra $\mathfrak{g}(A)$, with $d_{q}$ being $\mathfrak{g}(A)$-module homomorphisms, so that $H_{q}(G, V)$ are modules over $\mathfrak{g}(A)$.

Let $I$ be an ideal of $G$ and $L=G / I$. We define a filtration $\left\{K_{p}=K_{p} C\right\}$ of the complex $(C, d)$ by

$$
K_{p} C_{p+q}=\left\{g_{1} \wedge g_{2} \wedge \cdots \wedge g_{p+q} \otimes v \mid g_{i} \in I \text { for } p+1 \leq i \leq p+q\right\} .
$$

This gives rise to a spectral sequence $\left\{E_{p, q}^{r}, d_{r}: E_{p, q}^{r} \rightarrow E_{p-r, q+r-1}^{r}\right\}$ such that $E_{p, q}^{2} \cong H_{p}\left(L, H_{q}(I, V)\right)$ [H-S, Mc, Mo-T]. The terms $E_{p, q}^{r}$ are determined by

$$
E_{p, q}^{r+1}=\operatorname{Ker}\left(d_{r}: E_{p, q}^{r} \rightarrow E_{p-r, q+r-1}^{r}\right) / \operatorname{Im}\left(d_{r}: E_{p+r, q-r+1}^{r} \rightarrow E_{p, q}^{r}\right)
$$

with boundary homomorphism $d_{r+1}: E_{p, q}^{r} \rightarrow E_{p-r-1, q+r}^{r}$. For each $p, q$, the modules $E_{p, q}^{r}$ become stable for $r>\max (p, q+1)$, and the stable module is denoted by $E_{p, q}^{\infty}$. The spectral sequence $\left\{E_{p, q}^{r}, d_{r}\right\}$ converges to $H(G, V)$ in the following sense:

$$
H_{n}(G, V)=\bigoplus_{p+q=n} E_{p, q}^{\infty}
$$

When we have a convergent spectral sequence $\left\{E_{p, q}^{r}, d_{r}\right\}$ as above, we have the following exact sequence [C-E]:

$$
H_{2} \rightarrow E_{2,0}^{2} \rightarrow E_{0,1}^{2} \rightarrow H_{1} \rightarrow E_{1,0}^{2} \rightarrow 0 .
$$

In the above case, the following sequence is exact:

$$
\begin{aligned}
H_{2}(G, V) & \rightarrow H_{2}\left(L, H_{0}(I, V)\right) \rightarrow H_{0}\left(L, H_{1}(I, V)\right) \\
& \rightarrow H_{1}(G, V) \rightarrow H_{1}\left(L, H_{0}(I, V)\right) \rightarrow 0 .
\end{aligned}
$$

This sequence is called the Hochschild-Serre five term exact sequence. Ta summarize:

Theorem 3.1. Let $G$ be a Lie algebra and $V$ be a module over $G$. Let $I$ be an ideal of $G$ and let $L=G / I$. Then there exists a spectral sequence $\left\{E_{p, q}^{r}, d_{r}: E_{p, q}^{r} \rightarrow E_{p-r, q+r-1}^{r}\right\}$ converging to $H_{*}(G, V)$ such that

$$
E_{p, q}^{2} \cong H_{p}\left(L, H_{q}(I, V)\right) \text {. }
$$

Moreover, the following sequence is exact:

$$
\begin{aligned}
H_{2}(G, V) & \rightarrow H_{2}\left(L, H_{0}(I, V)\right) \rightarrow H_{0}\left(L, H_{1}(I, V)\right) \\
& \rightarrow H_{1}(G, V) \rightarrow H_{1}\left(L, H_{0}(I, V)\right) \rightarrow 0 .
\end{aligned}
$$

As an application of Theorem 3.1, we have the following lemma. 
Lemma 3.2. Let $\mathfrak{g}(A)$ be a Kac-Moody Lie algebra and let $G=\bigoplus_{n \geq 1} G_{n}$ be the free Lie algebra generated by a $\mathfrak{g}(A)$-module $G_{1}=V$. Let $I=\bigoplus_{n \geq m} I_{n}$ be the graded ideal of $G$ generated by the $\mathfrak{g}(A)$-submodule $I_{m}$ of $G_{m}$ for $m \geq 2$ and let $L=G / I$. Then we have an isomorphism of $\mathfrak{g}(A)$-modules

$$
H_{2}(L) \cong I_{m} \text {. }
$$

Proof. Note that $L=\bigoplus_{n \geq 1} L_{n}$ is also a graded Lie algebra generated by the subspace $L_{1}=G_{1}$. For the trivial module $\mathbf{C}$, the Hochschild-Serre five term exact sequence becomes

$$
H_{2}(G) \rightarrow H_{2}(L) \rightarrow H_{0}\left(L, H_{1}(I)\right) \rightarrow H_{1}(G) \rightarrow H_{1}(L) \rightarrow 0 .
$$

Since

$$
H_{1}(L) \cong \mathbf{C} \otimes L /[L, L] \cong L_{1} \text { and } H_{1}(G) \cong \mathbf{C} \otimes G /[G, G] \cong G_{1},
$$

the homomorphism $H_{1}(G) \rightarrow H_{1}(L)$ is an isomorphism. Since $G$ is free, by definition, $H_{2}(G)=0$. Therefore we have

$$
\begin{aligned}
H_{2}(L) & \cong H_{0}\left(L, H_{1}(I)\right) \cong H_{0}(L, I /[I, I]) \cong(I /[I, I]) / L \cdot(I /[I, I]) \\
& \cong(I /[I, I])_{m} \cong I_{m} .
\end{aligned}
$$

\section{The Kostant formula for Kac-Moody Lie algebras}

Let $A$ be an $n \times n$ symmetrizable generalized Cartan matrix and let $\mathfrak{g}(A)$ be the Kac-Moody Lie algebra with Cartan matrix $A$. Lat $\Delta \subset \mathfrak{h}^{*}$ be the root system of $\mathfrak{g}(A)$ and denote by $\Delta^{+}$(respectively, $\Delta^{-}$) the set of positive (respectively, negative) roots of $\mathfrak{g}(A)$. Then the triangular decomposition becomes $\mathfrak{g}(A)=\mathfrak{n}^{-} \oplus \mathfrak{h} \oplus \mathfrak{n}^{+}$, where $\mathfrak{n}^{ \pm}=\bigoplus_{\alpha \in \Delta^{ \pm}} \mathfrak{g}_{\alpha}$. Let $r_{i}$ be the reflection on $\mathfrak{h}^{*}$ determined by $\alpha_{i}$ for $i=1, \ldots, n$, and let $W$ be the Weyl group of $\mathfrak{g}(A)$ generated by the $r_{1}, \ldots, r_{n}$. The expression $w=r_{i_{1}} \cdots r_{i_{s}} \in W$ is called reduced if $s$ is minimal possible among all representations of $w \in W$ as a product of the $r_{i}$. In this case, $s$ is called the length of $w$ and is denoted by $l(w)$.

Let $S=\{1, \ldots, s\}$ be a subset of $N=\{1, \ldots, n\}$. Consider the subalgebra $\mathfrak{g} S$ of $\mathfrak{g}(A)$ generated by the elements $e_{i}, f_{i}(i=1, \ldots, s)$ and $\mathfrak{h}$. Denote by $\Delta_{S}^{+}$the set of positive roots generated by $\alpha_{1}, \ldots, \alpha_{s}$, and let $\Delta_{S}^{-}=-\Delta_{S}^{+}$. Then $\mathfrak{g}_{S}$ has a triangular decomposition $\mathfrak{g}_{S}=\mathfrak{n}_{S}^{-} \oplus \mathfrak{h} \oplus \mathfrak{n}_{s}^{+}$, where $\mathfrak{n}_{S}^{ \pm}=\bigoplus_{\alpha \in \Delta_{S}^{ \pm}} \mathfrak{g}_{\alpha}$, and $\Delta_{S}=\Delta_{S}^{+} \cup \Delta_{S}^{-}$is the root system of $\mathfrak{g}_{S}$. Let $\Delta^{ \pm}(S)=\Delta^{ \pm} \backslash \Delta_{S}^{ \pm}$and let $\mathfrak{n}^{ \pm}(S)=\bigoplus_{\alpha \in \Delta^{ \pm}(S)} \mathfrak{g}_{\alpha}$. Then

$$
\mathfrak{g}(A)=\mathfrak{n}^{-}(S) \oplus \mathfrak{g}_{S} \oplus \mathfrak{n}^{+}(S) .
$$

Note that $\mathfrak{n}^{ \pm}(S)$ are $\mathfrak{g}_{S}$-modules via adjoint action. Let $W_{S}$ be the Weyl group of $\mathfrak{g}_{S}$ generated by $r_{1}, \ldots, r_{s}$, and let

$$
W(S)=\left\{w \in W \mid w \Delta^{-} \cap \Delta^{+} \subset \Delta^{+}(S)\right\}=\left\{w \in W \mid \Phi_{w} \subset \Delta^{+}(S)\right\},
$$

where $\Phi_{w}=\left\{\alpha \in \Delta^{+} \mid w^{-1}(\alpha)<0\right\}$.

For $\lambda \in \mathfrak{h}^{*}$, we denote by $\widetilde{V}(\lambda)$ the irreducible highest weight module over $\mathfrak{g}(A)$ and $V(\lambda)$ the irreducible highest weight module over $\mathfrak{g}_{S}$. Then the structure of the homology modules $H_{*}\left(\mathfrak{n}^{-}(S), \widetilde{V}(\lambda)\right)$ is determined by the following theorem. 
Theorem 4.1 [Liu].

$$
H_{j}\left(\mathfrak{n}^{-}(S), \widetilde{V}(\lambda)\right) \cong \bigoplus_{\substack{w \in W(S) \\ l(w)=j}} V(w(\lambda+\rho)-\rho)
$$

Remark 4.2. This formula was first introduced by Kostant for finite dimensional complex semisimple Lie algebras [Ko]. In [G-L], Garland and Lepowsky proved this formula for symmetrizable Kac-Moody Lie algebras under the condition that $S$ is of finite type. In [Liu], Liu proved this formula for symmetrizable Kac-Moody Lie algebras without assuming that $S$ is of finite type. We will call the formula (4.2) the Kostant formula.

The following lemma is very useful in the actual computation.

Lemma 4.3. Suppose $w=w^{\prime} r_{j}$ and $l(w)=l\left(w^{\prime}\right)+1$. Then $w \in W(S)$ if and only if $w^{\prime} \in W(S)$ and $w^{\prime}\left(\alpha_{j}\right) \in \Delta^{+}(S)$.

Proof. By definition, $w \in W(S)$ if and only if $\Phi_{w}=\left\{\alpha \in \Delta^{+} \mid w^{-1}(\alpha)<0\right\} \subset$ $\Delta^{+}(S)$. But $\Phi_{w}=\Phi_{w^{\prime}} \cup\left\{w^{\prime}\left(\alpha_{j}\right)\right\}$. Thus $\Phi_{w} \subset \Delta^{+}(S)$ if and only if $\Phi_{w^{\prime}} \subset$ $\Delta^{+}(S)$ and $w^{\prime}\left(\alpha_{j}\right) \in \Delta^{+}(S)$, which is equivalent to saying that $w^{\prime} \in W(S)$ and $w^{\prime}\left(\alpha_{j}\right) \in \Delta^{+}(S)$.

Now let $A=\left(a_{i j}\right)_{i, j=0,1, \ldots, l}$ be a generalized Cartan matrix of affine type and let $\tilde{A}=\left(a_{i j}\right)_{i, j=-1,0,1, \ldots, l}$ be a generalized Cartan matrix of Lorentzian type as defined in $\S 1$. We have seen that the Kac-Moody Lie algebra $\mathfrak{g}(\widetilde{A})$ can be realized as the minimal graded Lie algebra $L=\bigoplus_{n \in Z} L_{n}$ with the local part $V+\mathfrak{g}(A)+V^{*}$, where $V=V\left(-\alpha_{-1}\right)$ is the basic representation of the affine Kac-Moody Lie algebra $\mathfrak{g}(A)$. Let $L_{ \pm}=\bigoplus_{n \geq 1} L_{ \pm n}$. Then $L_{ \pm}$coincides with $\mathfrak{n}^{ \pm}(S)$ for the set $S=\{0,1, \ldots, l\}$. Thus the Kostant formula enables us to compute the homology modules of the Lie algebra $L_{ \pm}$with coefficients in the trivial module $\mathbf{C}$ :

$$
H_{j}\left(L_{-}\right) \cong \bigoplus_{\substack{w \in W(S) \\ l(w)=j}} V(w \rho-\rho)
$$

Example 4.4. Let

$$
\widetilde{A}=\left(a_{i j}\right)_{i, j=-1,0,1}=\left(\begin{array}{rrr}
2 & -1 & 0 \\
-1 & 2 & -2 \\
0 & -2 & 2
\end{array}\right)
$$

be a generalized Cartan matrix of hyperbolic type. We realize the corresponding hyperbolic Kac-Moody Lie algebra $H A_{1}^{(1)}$ as the minimal graded Lie algebra $L=\bigoplus_{n \in Z} L_{n}$ with local part $V+\mathfrak{g}(A)+V^{*}$, where $V=V\left(-\alpha_{-1}\right)$ is the basic representation of the affine Kac-Moody Lie algebra $A_{1}^{(1)}$. Let $L_{ \pm}=\bigoplus_{n \geq 1} L_{ \pm n}$ and let $S=\{0,1\}$. We will compute some of the homology modules of the Lie algebra $L_{ \pm}$with coefficients in the trivial module $\mathbf{C}$ using the Kostant formula.

For $j=1$, the only element in $W(S)$ of length 1 is $r_{-1}$, and $r_{-1} \rho-\rho=$ $-\alpha_{-1}$. Thus $H_{1}\left(L_{-}\right) \cong V\left(-\alpha_{-1}\right)$. For $j=2$, by Lemma 4.3 , we have only to consider the element $r_{-1} r_{0}$. Since $r_{-1}\left(\alpha_{0}\right)=\alpha_{-1}+\alpha_{0} \in \Delta^{+}(S), r_{-1} r_{0} \in W(S)$ by Lemma 4.3. We have $r_{-1} r_{0} \rho-\rho=-2 \alpha_{-1}-\alpha_{0}$, and hence $H_{2}\left(L_{-}\right) \cong$ 
$V\left(-2 \alpha_{-1}-\alpha_{0}\right)$. For $j=3$, by Lemma 4.3 , we need to check the elements $r_{-1} r_{0} r_{-1}$ and $r_{-1} r_{0} r_{1}$. Since

$$
r_{-1} r_{0}\left(\alpha_{1}\right)=2 \alpha_{-1}+2 \alpha_{0}+\alpha_{1} \in \Delta^{+}(S),
$$

we have $r_{-1} r_{0} r_{1} \in W(S)$. But $r_{-1} r_{0}\left(\alpha_{-1}\right)=\alpha_{0} \notin \Delta^{+}(S)$, hence $r_{-1} r_{0} r_{-1} \notin$ $W(S)$. By an easy calculation, we get

$$
r_{-1} r_{0} r_{1} \rho-\rho=-4 \alpha_{-1}-3 \alpha_{0}-\alpha_{1} .
$$

Therefore

$$
H_{3}\left(L_{-}\right) \cong V\left(-4 \alpha_{-1}-3 \alpha_{0}-\alpha_{1}\right) .
$$

Continuing this process, we obtain

$$
\begin{aligned}
& H_{4}\left(L_{-}\right) \cong V\left(-7 \alpha_{-1}-6 \alpha_{0}-3 \alpha_{1}\right), \\
& H_{5}\left(L_{-}\right) \cong V\left(-10 \alpha_{-1}-10 \alpha_{0}-5 \alpha_{1}\right) \oplus V\left(-11 \alpha_{-1}-10 \alpha_{0}-6 \alpha_{1}\right), \text { etc. }
\end{aligned}
$$

\section{HoMOlogicAl STUDY OF THE GRADED LIE ALGEBRAS}

Let $G=\bigoplus_{n>1} G_{n}$ be the free Lie algebra generated by the subspace $G_{1}$, and let $I=\bigoplus_{n \geq m} \bar{I}_{n}$ be the graded ideal of $G$ generated by the subspace $I_{m}$ for $m \geq 2$. Consider the quotient Lie algebra $L=G / I$. Then $L=\bigoplus_{n>1} L_{n}$ is also a graded Lie algebra generated by the subspace $L_{1}=G_{1}$. Let $J=I /[I, I]$. Since $I$ is an ideal of $G, L$ acts on $J=I /[I, I]$ via adjoint action, and thus $J$ becomes an $L$-module generated by the subspace $J_{m}$. Note that, as vector spaces, $J_{n} \cong I_{n}$ for $m \leq n<2 m$.

Suppose that $G_{1}$ and $I_{m}$ are modules over a Kac-Moody Lie algebra $\mathfrak{g}(A)$. Then each homogeneous subspace $G_{n}$ has a $\mathfrak{g}(A)$-module structure such that $x \cdot[v, w]=[x \cdot v, w]+[v, x \cdot w]$ for $x \in \mathfrak{g}(A), v \in G_{1}, w \in G_{n-1}$. Then $\mathfrak{g}(A)$-module structure on the homogeneous spaces $I_{n}$ is given similarly. We also have the induced $\mathfrak{g}(A)$-module structure on the homogeneous subspaces $L_{n}$ and $J_{n}$.

Now consider the exact sequence of $L$-modules

$$
0 \rightarrow K \rightarrow U(L) \otimes J_{m} \stackrel{\psi}{\rightarrow} J \rightarrow 0,
$$

where $\psi$ is the usual bracket mapping, and $K$ is the kernel of $\psi$. This produces a long exact sequence

$$
\begin{aligned}
\cdots & \rightarrow H_{1}(L, K) \rightarrow H_{1}\left(L, U(L) \otimes J_{m}\right) \rightarrow H_{1}(L, J) \rightarrow H_{0}(L, K) \\
& \rightarrow H_{0}\left(L, U(L) \otimes J_{m}\right) \rightarrow H_{0}(L, J) \rightarrow 0 .
\end{aligned}
$$

Since $U(L) \otimes J_{m}$ is free over $L$, i.e., over $U(L)$, we have

$$
H_{j}\left(L, U(L) \otimes J_{m}\right)=0 \text { for } j \geq 1
$$

[J1, J2]. By definition, $H_{0}(L, J) \cong J /[L, J] \cong J_{m}$ and

$$
H_{0}\left(L, U(L) \otimes J_{m}\right) \cong U(L) \otimes J_{m} / L\left(U(L) \otimes J_{m}\right) \cong\left(U(L) \otimes J_{m}\right)_{m} \cong J_{m} .
$$

Thus from the long exact sequence we get a $\mathfrak{g}(A)$-module isomorphism

$$
H_{1}(L, J) \cong H_{0}(L, K) \cong K / L \cdot K \text {. }
$$

On the other hand, using the Poincaré-Birkhoff-Witt theorem, we can prove the following lemma (e.g., [Kac2, Exercise 9.13; Kang, Lemma 4.5]). 
Lemma 5.1. There is an exact sequence of L-modules

$$
0 \rightarrow J \stackrel{\alpha}{\rightarrow} U(L) \otimes G_{1} \stackrel{\beta}{\rightarrow} U(L) \stackrel{\vartheta}{\rightarrow} \mathbf{C} \rightarrow 0 .
$$

Proof. Consider the homomorphisms

$$
\alpha: J=I /[I, I] \rightarrow U_{0}(G) / I U_{0}(G) \text { and } \beta: U_{0}(G) / I U_{0}(G) \rightarrow U(L)
$$

induced by the natural injection $I \rightarrow U_{0}(G)$ and by the canonical surjection $G \rightarrow L$, respectively. Define a homomorphism $\gamma: U(L) \rightarrow \mathbf{C}$ by $\left.\gamma\right|_{\mathbf{C}}=$ id and $\gamma\left(U_{0}(L)\right)=0$.

By the Poincaré-Birkhoff-Witt theorem, we have $I \cap I U_{0}(G)=[I, I]$, and hence $\operatorname{Ker} \alpha=0$. It is clear that $\operatorname{Im} \alpha=I+I U_{0}(G) / I U_{0}(G)$. Since $I U(G) \subset$ $U_{0}(G)$,

$$
\operatorname{Ker} \beta=U_{0}(G) \cap I U(G) / I U_{0}(G)=I U(G) / I U_{0}(G) .
$$

Since $U(G)=\mathbf{C}+U_{0}(G), I U(G)=I+I U_{0}(G)$. Thus $\operatorname{Ker} \beta=I+I U_{0}(G)=$ $\operatorname{Im} \alpha$. Finally, it is obvious that $\operatorname{Im} \beta=U_{0}(L)=\operatorname{Ker} \gamma$. Therefore we have an exact sequence

$$
0 \rightarrow J \stackrel{\alpha}{\rightarrow} U_{0}(G) / I U_{0}(G) \stackrel{\beta}{\rightarrow} U(L) \stackrel{\gamma}{\rightarrow} \mathbf{C} \rightarrow 0 .
$$

Let $\mathfrak{B}_{0}=\left\{u_{i} \mid i \in \Omega\right\}$ be a basis of $I$ and let

$$
\mathfrak{B}=\left\{u_{i} \mid i \in \Omega\right\} \cup\left\{u_{j}^{\prime} \mid j \in \Omega^{\prime}\right\}
$$

be a basis of $G$ extending $\mathfrak{B}_{0}$. By the Poincaré-Birkhoff-Witt theorem, we have a basis of $U(G)$ consisting of the elements of the form

$$
u_{i_{1}} u_{i_{2}} \cdots u_{i_{s}} u_{j_{1}}^{\prime} u_{j_{2}}^{\prime} \cdots u_{j_{t}}^{\prime}
$$

with $i_{1} \leq \cdots \leq i_{s}$ and $j_{1} \leq \cdots \leq j_{t}$. Note that $U(L) \cong U(G) / I U(G)$. We define a linear map $\eta: U(L) \otimes_{U(G)} U_{0}(G) \rightarrow U_{0}(G) / I U_{0}(G)$ by

$$
\eta((u+I U(G)) \otimes v)=u v+I U_{0}(G)
$$

for $u \in U(G), v \in U_{0}(G)$. Using the PBW basis (5.4), it is easy to show that $\eta$ is an isomorphism. Hence we have the following isomorphisms

$$
U_{0}(G) / I U_{0}(G) \cong U(L) \otimes_{U(G)} U_{0}(G) \cong U(L) \otimes_{U(G)}\left(U(G) \otimes G_{1}\right) \cong U(L) \otimes G_{1} .
$$

Therefore we get the desired exact sequence.

Theorem 5.2. There is an isomorphism of $\mathfrak{g}(A)$-modules

$$
H_{j}(L, J) \cong H_{j+2}(L) \text { for } j \geq 1 .
$$

Proof. We split the exact sequence (5.3) into the following exact sequences:

$$
0 \rightarrow J \stackrel{\alpha}{\rightarrow} U(L) \otimes G_{1} \stackrel{\beta}{\rightarrow} \operatorname{Im} \beta \rightarrow 0,
$$

and

$$
0 \rightarrow \operatorname{Im} \beta=\operatorname{Ker} \gamma \rightarrow U(L) \rightarrow \operatorname{Coker} \beta \rightarrow 0 .
$$

Then we get long exact sequences:

$$
\begin{aligned}
\cdots & \rightarrow H_{j+1}(L, J) \rightarrow H_{j+1}\left(L, U(L) \otimes G_{1}\right) \rightarrow H_{j+1}(L, \operatorname{Im} \beta) \\
& \rightarrow H_{j}(L, J) \rightarrow H_{j}\left(L, U(L) \otimes G_{1}\right) \rightarrow H_{j}(L, \operatorname{Im} \beta) \rightarrow \cdots,
\end{aligned}
$$


and

$$
\begin{aligned}
\cdots & \rightarrow H_{j+2}(L, \operatorname{Im} \beta) \rightarrow H_{j+2}(L, U(L)) \rightarrow H_{j+2}(L, \operatorname{Coker} \beta) \\
& \rightarrow H_{j+1}(L, \operatorname{Im} \beta) \rightarrow H_{j+1}(L, U(L)) \rightarrow H_{j+1}(L, \operatorname{Coker} \beta) \rightarrow \cdots .
\end{aligned}
$$

Since $U(L)$ and $U(L) \otimes G_{1}$ are free over $U(L)$, we have

$$
H_{k}(L, U(L))=H_{k}\left(L, U(L) \otimes G_{1}\right)=0 \text { for } k \geq 1 .
$$

Thus we get

$$
H_{j}(L, J) \cong H_{j+1}(L, \operatorname{Im} \beta) \cong H_{j+2}(L, \operatorname{Coker} \beta) \text { for } j \geq 1 .
$$

But since $\gamma$ is surjective, $\operatorname{Coker} \beta=U(L) / \operatorname{Ker} \gamma \cong \mathbf{C}$. Therefore we get the desired isomorphism.

In particular, $H_{1}(L, J) \cong H_{3}(L)$. Therefore combining with (5.2) yields

$$
K / L \cdot K \cong H_{3}(L) \text {. }
$$

Note that $H_{3}(L)$ has a graded module structure over $\mathfrak{g}(A)$ induced by that of $L$.

Theorem 5.3. Let $t$ be the smallest homogeneous degree of $H_{3}(L)$. Then $K_{j}=0$ for $j<t$ and $K_{t} \cong H_{3}(L)_{t}$. In particular, we have

$$
K_{m+1} \cong H_{3}(L)_{m+1} \text {. }
$$

Proof. Note that $K_{m}=0$. Thus $H_{3}(L)_{m}=(K / L \cdot K)_{m}=0$ and hence $t \geq$ $m+1$. Now for $m \leq j<t$, assume inductively that $K_{m}=\cdots=K_{j-1}=0$. Then

$$
0=H_{3}(L)_{j} \cong(K / L \cdot K)_{j} \cong K_{j} /\left(\sum_{i=m}^{j-1} L_{j-i} \cdot K_{i}\right) \cong K_{j},
$$

proving the first assertion. Similarly, since $K_{m}=\cdots=K_{t-1}=0$, we have

$$
H_{3}(L)_{t} \cong(K / L \cdot K)_{t} \cong K_{t} /\left(\sum_{i=m}^{t-1} L_{t-i} \cdot K_{i}\right) \cong K_{t} .
$$

Theorem 5.4. (a) For $m \leq j<\min (2 m, t)$, we have

$$
I_{j} \cong \underbrace{G \otimes \cdots \otimes G_{1}}_{(j-m) \text { times }} \otimes I_{m} .
$$

(b) If $t<2 m$, we have

$$
I_{t} \cong \underbrace{G_{1} \otimes \cdots \otimes G_{1}}_{(t-m) \text { times }} \otimes I_{m} / H_{3}(L)_{t} .
$$

In particular,

$$
I_{m+1} \cong G_{1} \otimes I_{m} / H_{3}(L)_{m+1} .
$$

Proof. (a) Since $K_{j}=0$ for $j<t$, we have

$$
I_{j} \cong J_{j} \cong\left(U(L) \otimes I_{m}\right)_{j} / K_{j}=\left(U(L) \otimes I_{m}\right)_{j} \cong U(L)_{j-m} \otimes I_{m} .
$$

Since $m \leq j<\min (2 m, t)$, we have $j-m<m$. Thus $L_{k} \cong G_{k}$ for $1 \leq k \leq$ $j-m$, which implies $U(L)_{j-m} \cong U(G)_{j-m}$. Since $G$ is the free Lie algebra 
generated by the subspace $G_{1}$, the universal enveloping algebra of $G$ is the tensor algebra on $G_{1}$. Hence we get

$$
U(G)_{j-m} \cong \underbrace{G_{1} \otimes \cdots \otimes G_{1}}_{(j-m) \text { times }} .
$$

Therefore

$$
I_{j} \cong \underbrace{G_{1} \otimes \cdots \otimes G_{1}}_{(j-m) \text { times }} \otimes I_{m}
$$

(b) Since $t<2 m$, we have

$$
I_{t} \cong J_{t} \cong\left(U(L) \otimes I_{m}\right)_{t} / K_{t} \cong\left(U(L)_{t-m} \otimes I_{m}\right) / K_{t} .
$$

By Theorem 5.3, $K_{t} \cong H_{3}(L)_{t}$, and the argument of (a) gives

$$
U(L)_{t-m} \cong U(G)_{t-m} \cong \underbrace{G_{1} \otimes \cdots \otimes G_{1}}_{(t-m) \text { times }} .
$$

Therefore

$$
I_{t} \cong \underbrace{G_{1} \otimes \cdots \otimes G_{1}}_{(t-m) \text { times }} \otimes I_{m} / H_{3}(L)_{t}
$$

In particular,

$$
I_{m+1} \cong\left(G_{1} \otimes I_{m}\right) / H_{3}(L)_{m+1} .
$$

Now we study the homogeneous spaces $I_{j}$ of $I$ for arbitrary $j \geq m$. We repeat the above process under the following setting. For $j \geq m$, let $I^{(j)}=$ $\sum_{n \geq j} I_{n}$. Since $I_{m}$ generates the ideal $I$, we have

$$
I_{m+1}=\left(\operatorname{ad} G_{1}\right)\left(I_{m}\right), \ldots, I_{n}=\left(\operatorname{ad} G_{1}\right)^{n-m}\left(I_{m}\right)=\left(\operatorname{ad} G_{1}\right)\left(I_{n-1}\right) .
$$

Thus $I^{(j)}$ is an ideal of $G$ generated by the subspace $I_{j}$. Consider the quotient Lie algebra $L^{(j)}=G / I^{(j)}$. Thus $L=L^{(m)}$ in this notation. Set $J^{(j)}=$ $I^{(j)} /\left[I^{(j)}, I^{(j)}\right]$. Then $J^{(j)}$ is an $L^{(j)}$-module generated by the subspace $J_{j}^{(j)}$. Note that $J_{n}^{(j)} \cong I_{n}^{(j)}=I_{n}$ for $j \leq n<2 j$. Considering the exact sequences

$$
0 \rightarrow K^{(j)} \rightarrow U\left(L^{(j)}\right) \otimes J_{j}^{(j)} \stackrel{\psi}{\rightarrow} J^{(j)} \rightarrow 0,
$$

and

$$
0 \rightarrow J^{(j)} \stackrel{\alpha}{\rightarrow} U\left(L^{(j)}\right) \otimes G_{1} \stackrel{\beta}{\rightarrow} U\left(L^{(j)}\right) \stackrel{\vartheta}{\rightarrow} \mathbf{C} \rightarrow 0,
$$

the same homological argument shows that the structure of $H_{3}\left(L^{(j)}\right)$ determines the $\mathfrak{g}(A)$-module structure of the homogeneous spaces $I_{n}$ for $j+1 \leq n<$ $\min \left(2 j, t^{(j)}\right)$, where $t^{(j)}$ is the smallest homogeneous degree of $H_{3}\left(L^{(\bar{j})}\right)$. In particular,

$$
I_{j+1} \cong\left(G_{1} \otimes I_{j}\right) / H_{3}\left(L^{(j)}\right)_{j+1} .
$$

Therefore we now have an inductive algorithm to determine the $\mathfrak{g}(A)$-module structure of the homogeneous subspaces of the graded ideal $I$ by studying the homology modules $H_{3}\left(L^{(j)}\right)$ for $j \geq m$.

Suppose we have determined the structure of homology modules $H_{*}\left(L^{(j-1)}\right)$. We define the subspace $N^{(j-1)}$ of $L^{(j)}$ by $N^{(j-1)}=I^{(j-1)} / I^{(j)}$. Then $N^{(j-1)}$ is an abelian ideal of $L^{(j)}$ and $L^{(j)} / N^{(j-1)}$ is isomorphic to $L^{(j-1)}$. Then from 
Theorem 3.1, there exists a spectral sequence $\left\{E_{p, q}^{r}, d_{r}: E_{p, q}^{r} \rightarrow E_{p-r, q+r-1}^{r}\right\}$ converging to $H_{*}\left(L^{(j)}\right)$ such that

$$
E_{p, q}^{2} \cong H_{p}\left(L^{(j-1)}, H_{q}\left(N^{(j-1)}\right)\right) .
$$

In particular, from (3.4), we have

$$
H_{3}\left(L^{(j)}\right) \cong E_{3,0}^{\infty} \oplus E_{2,1}^{\infty} \oplus E_{1,2}^{\infty} \oplus E_{0,3}^{\infty} .
$$

Thus to study the structure of $H_{3}\left(L^{(j)}\right)$, we need to determine the boundary maps $d_{r}: E_{p, q}^{r} \rightarrow E_{p-r, q+r-1}^{r}$. The maps $d_{r}$ are $\mathfrak{g}(A)$-module homomorphisms since they are induced by the $\mathfrak{g}(A)$-module homomorphisms $d_{q}$ defined by (3.1).

Since $L^{(j-1)}$ acts on $N^{(j-1)}$ trivially, we have

$$
H_{p}\left(L^{(j-1)}, H_{q}\left(N^{(j-1)}\right)\right) \cong H_{p}\left(L^{(j-1)}\right) \otimes H_{q}\left(N^{(j-1)}\right) .
$$

Note that $N^{(j-1)} \cong I_{j-1}$ as $\mathfrak{g}(A)$-modules and that $N^{(j-1)}$ is an abelian Lie algebra. Thus, as $\mathfrak{g}(A)$-modules, $H_{q}\left(N^{(j-1)}\right) \cong \Lambda^{q}\left(I_{j-1}\right)$. Therefore we have

$$
E_{p, q}^{2} \cong H_{p}\left(L^{(j-1)}\right) \otimes \Lambda^{q}\left(I_{j-1}\right) \text {. }
$$

In the next section, using the homological approach developed here, together with the representation theory of affine Kac-Moody Lie algebras, we will determine some of the boundary homomorphisms and deduce some new structural information on the Kac-Moody Lie algebras of Lorentzian type.

\section{THE STRUCTURE OF THE MAXIMAL GRADED IDEAL}

In this section, we study the structure of the maximal graded ideal $I$. We know that the ideal $I_{-}$of $G_{-}$is generated by the homogeneous subspace $I_{-2}$ and hence we may write $I_{-}=I_{-}^{(2)}$ following the notation introduced in the previous section. Similarly, for $j \geq 2$, we write $I_{-}^{(j)}=\sum_{n \geq j} I_{-n}, L_{-}^{(j)}=G / I_{-}^{(j)}$, and $N_{-}^{(j)}=I_{-}^{(j)} / I_{-}^{(j+1)}$.

\section{Lemma 6.1.}

$$
I_{-2} \cong V\left(-2 \alpha_{-1}-\alpha_{0}\right) .
$$

Proof. Since $G_{-}$is free and $I_{-}$is generated by the subspace $I_{-2}$, from the Hochschild-Serre five term exact sequence, we see that $I_{-2} \cong H_{2}\left(L_{-}\right)$(Lemma 3.2). By the Kostant formula (see the computations at the end of $\S 4$ ), we have

$$
H_{2}\left(L_{-}\right) \cong \sum_{\substack{w \in W(S) \\ l(w)=2}} V(w \rho-\rho) \cong V\left(r_{-1} r_{0} \rho-\rho\right)=V\left(-2 \alpha_{-1}-\alpha_{0}\right) .
$$

By the homological theory developed in the previous section, we have in general that

$$
I_{-(j+1)} \cong V \otimes I_{-j} / H_{3}\left(L_{-}^{(j)}\right)_{-(j+1)} \quad \text { for } j \geq 2 .
$$

When $j=2, L_{-}^{(2)}$ coincides with the subalgebra $\mathfrak{n}^{-}(S)$ for $S=\{0,1, \ldots, l\}$, and therefore we can compute $H_{3}\left(L_{-}^{(2)}\right)$ using the Kostant formula. For instance, in the case of $H A_{1}^{(1)}$, we have $H_{3}\left(L_{-}^{(2)}\right) \cong V\left(-4 \alpha_{-1}-3 \alpha_{0}-\alpha_{1}\right)$. Thus $H_{3}\left(L_{-}^{(2)}\right)_{-3}=0$, and hence we obtain

$$
I_{-3} \cong V \otimes I_{-2} / H_{3}\left(L_{-}^{(2)}\right)_{-3} \cong V \otimes I_{-2} .
$$


So we can easily determine the structure of the subspace $I_{-3}$ using the Kostant formula. However, for $j \geq 3$, we cannot use the Kostant formula to compute the homology module $H_{3}\left(L_{-}^{(j)}\right)$. We study the higher levels by developing some techniques from the Hochschild-Serre spectral sequences.

Theorem 6.2. Let $\mathfrak{g}(A)$ be an affine Kac-Moody Lie algebra, $V$ be its basic representation, and let $G, I$, and $L$ be as defined in $\S 1$. Then we have the following information on the $\mathfrak{g}(A)$-module structure of the maximal graded ideal $I$.

$$
\begin{aligned}
& H A_{1}^{(1)}: \quad I_{-3} \cong V \otimes I_{-2}, \\
& I_{-4} \cong V \otimes I_{-3} /\left(V\left(-4 \alpha_{-1}-3 \alpha_{0}-\alpha_{1}\right) \oplus S^{2}\left(I_{-2}\right)\right), \\
& I_{-5} \cong V \otimes I_{-4} / V \otimes \Lambda^{2}\left(I_{-2}\right) \text {; } \\
& H A_{2}^{(2)}: \quad I_{-3} \cong V \otimes I_{-2} \text {, } \\
& I_{-4} \cong V \otimes I_{-3} / S^{2}\left(I_{-2}\right) \text {, } \\
& I_{-5} \cong V \otimes I_{-4} / V \otimes \Lambda^{2}\left(I_{-2}\right) \\
& H A_{l}^{(1)}, l \geq 2: \quad I_{-3} \cong V \otimes I_{-2} /\left(V\left(-3 \alpha_{-1}-2 \alpha_{0}-\alpha_{1}\right)\right. \\
& \left.\oplus V\left(-3 \alpha_{-1}-2 \alpha_{0}-\alpha_{l}\right)\right) \\
& I_{-4} \cong V \otimes I_{-3} / S^{2}\left(I_{-2}\right) \\
& H C_{2}^{(1)}, H D_{4}^{(3)}: \quad I_{-3} \cong V \otimes I_{-2} / V\left(-3 \alpha_{-1}-2 \alpha_{0}-\alpha_{1}\right) \text {, } \\
& I_{-4} \cong V \otimes I_{-3} / S^{2}\left(I_{-2}\right) \\
& H A_{2 l}^{(2)}, H D_{l+1}^{(2)}, l \geq 2: \quad I_{-3} \cong V \otimes I_{-2} \text {, } \\
& I_{-4} \cong V \otimes I_{-3} /\left(V\left(-4 \alpha_{-1}-3 \alpha_{0}-\alpha_{1}\right) \oplus S^{2}\left(I_{-2}\right)\right) ; \\
& H B_{l}^{(1)}, l \geq 3, H D_{l}^{(1)}, l \geq 4: \quad I_{-3} \cong V \otimes I_{-2} / V\left(-3 \alpha_{-1}-2 \alpha_{0}-\alpha_{2}\right) \text {; } \\
& H C_{l}^{(1)}, H A_{2 l-1}^{(2)}, l \geq 3, H F_{4}^{(1)}, H G_{2}^{(1)}, H E_{7}^{(1)}, H E_{8}^{(1)}, H E_{6}^{(2)} \text { : } \\
& I_{-3} \cong V \otimes I_{-2} / V\left(-3 \alpha_{-1}-2 \alpha_{0}-\alpha_{1}\right) \text {; } \\
& H E_{6}^{(1)}: \quad I_{-3} \cong V \otimes I_{-2} / V\left(-3 \alpha_{-1}-2 \alpha_{0}-\alpha_{6}\right) \text {. }
\end{aligned}
$$

Proof. The Kostant formula determines the $\mathfrak{g}(A)$-module structure of the subspace $I_{-3}$ for all cases. For higher levels, we consider the following four cases separately.

Case 1. $H A_{1}^{(1)}$.

By (6.2), to determine the structure of $I_{-4}$, we need to determine the structure of $H_{3}\left(L_{-}^{(3)}\right)_{-4}$. We consider the following short exact sequence

$$
0 \rightarrow N_{-}^{(2)} \rightarrow L_{-}^{(3)} \rightarrow L_{-}^{(2)} \rightarrow 0
$$

and the corresponding spectral sequence $\left\{E_{p, q}^{r}\right\}$ converging to $H_{*}\left(L_{-}^{(3)}\right)$ such that

$$
E_{p, q}^{2} \cong H_{p}\left(L_{-}^{(2)}\right) \otimes \Lambda^{q}\left(I_{-2}\right)
$$


We will compute $H_{3}\left(L_{-}^{(3)}\right)_{-4}$ from this sequence.

Let us start with the sequence

$$
0 \rightarrow E_{2,0}^{2} \stackrel{d_{2}}{\rightarrow} E_{0,1}^{2} \rightarrow 0 .
$$

Note that

$$
H_{1}\left(L_{-}^{(3)}\right) \cong L_{-}^{(3)} /\left[L_{-}^{(3)}, L_{-}^{(3)}\right] \cong L_{-1}=V .
$$

Since the spectral sequence converges to $H_{*}\left(L_{-}^{(3)}\right)$, we have

$$
H_{1}\left(L_{-}^{(3)}\right) \cong E_{1,0}^{\infty} \oplus E_{0,1}^{\infty} .
$$

But

$$
E_{1,0}^{\infty}=E_{1,0}^{2} \cong H_{1}\left(L_{-}^{(2)}\right) \cong L_{1}^{(2)} /\left[L_{-}^{(2)}, L_{-}^{(2)}\right] \cong L_{-1}=V,
$$

which implies $E_{0,1}^{\infty}=E_{0,1}^{3}=0$. Hence the homomorphism $d_{2}$ is surjective. Since $E_{2,0}^{2} \cong I_{-2}$ and $E_{0,1}^{2} \cong I_{-2}, d_{2}$ must be an isomorphism. Thus $E_{2,0}^{3}=$ 0 , and hence $E_{2,0}^{\infty}=0$.

Now consider the following sequence

$$
0 \rightarrow E_{3,0}^{2} \stackrel{d_{2}}{\rightarrow} E_{1,1}^{2} \rightarrow 0 .
$$

By the Kostant formula, we have

$$
E_{3,0}^{2} \cong H_{3}\left(L_{-}^{(2)}\right) \cong V\left(-4 \alpha_{-1}-3 \alpha_{0}-\alpha_{1}\right)
$$

and

$$
E_{1,1}^{2} \cong H_{1}\left(L_{-}^{(2)}\right) \otimes I_{-2} \cong V \otimes I_{-2} .
$$

Since $V \otimes I_{-2}$ is a direct sum of irreducible highest weight modules over $A_{1}^{(1)}$ of level 3 , by comparing the levels of both terms, we see that $d_{2}: E_{3,0}^{2} \rightarrow E_{1,1}^{2}$ is trivial. So $E_{3,0}^{3}=E_{3,0}^{2}$, and $E_{1,1}^{\infty}=E_{1,1}^{3}=E_{1,1}^{2} \cong V \otimes I_{-2}$. Since $I_{-}^{(3)}$ is generated by $I_{-3}$, by Lemma 3.2, and (6.3) give

$$
H_{2}\left(L_{-}^{(3)}\right) \cong I_{-3}=V \otimes I_{-2} .
$$

But we have

$$
H_{2}\left(L_{-}^{(3)}\right) \cong E_{2,0}^{\infty} \oplus E_{1,1}^{\infty} \oplus E_{0,2}^{\infty} .
$$

It follows that $E_{0,2}^{\infty}=E_{0,2}^{4}=0$. Hence we conclude either $E_{0,2}^{3}=0$ or the homomorphism $d_{3}: E_{3,0}^{3} \rightarrow E_{0,2}^{3}$ is surjective.

Assume first that $E_{0,2}^{3}=0$. This implies that $d_{3}: E_{3,0}^{3} \rightarrow E_{0,2}^{3}$ is trivial and that the homomorphism $d_{2}: E_{2,1}^{2} \rightarrow E_{0,2}^{2}$ is surjective in the sequence

$$
0 \rightarrow E_{4,0}^{2} \rightarrow E_{2,1}^{2} \rightarrow E_{0,2}^{2} \rightarrow 0 \text {. }
$$

Thus

$$
\begin{aligned}
E_{3,0}^{\infty} & =E_{3,0}^{4}=\operatorname{Ker}\left(d_{3}: E_{3,0}^{3} \rightarrow E_{0,2}^{3}\right) / \operatorname{Im}\left(d_{3}: 0 \rightarrow E_{3,0}^{3}\right) \\
& =E_{3,0}^{3}=E_{3,0}^{2} \cong V\left(-4 \alpha_{-1}-3 \alpha_{0}-\alpha_{1}\right) .
\end{aligned}
$$

By comparing levels, we see that $d_{2}: E_{4,0}^{2} \rightarrow E_{2,1}^{2}$ must be trivial. Note that $E_{0,2}^{2} \cong \Lambda^{2}\left(I_{-2}\right)$. Therefore $E_{4,0}^{3}=E_{4,0}^{2}$ and

$$
\begin{aligned}
E_{2,1}^{\infty} & =E_{2,1}^{3}=\operatorname{Ker}\left(d_{2}: E_{2,1}^{2} \rightarrow E_{0,2}^{2}\right) / \operatorname{Im}\left(d_{2}: E_{4,0}^{2} \rightarrow E_{2,1}^{2}\right) \\
& \cong \operatorname{Ker}\left(d_{2}: E_{2,1}^{2} \rightarrow E_{0,2}^{2}\right) .
\end{aligned}
$$


Since $d_{2}: E_{2,1}^{2} \rightarrow E_{0,2}^{2}$ is surjective, we have

$$
\Lambda^{2}\left(I_{-2}\right) \cong E_{0,2}^{2} \cong E_{2,1}^{2} / \operatorname{Ker} d_{2} \cong I_{-2} \otimes I_{-2} / \operatorname{Ker} d_{2} \text {. }
$$

Therefore $\operatorname{Ker} d_{2} \cong S^{2}\left(I_{-2}\right)$. Hence $E_{2,1}^{\infty} \cong S^{2}\left(I_{-2}\right)$.

If $E_{0,2}^{3}$ is nonzero and $d_{3}: E_{3,0}^{3} \rightarrow E_{0,2}^{3}$ is surjective, then since $E_{3,0}^{3}=E_{3,0}^{2}$ is irreducible, $d_{3}: E_{3,0}^{3} \rightarrow E_{0,2}^{3}$ is an isomorphism. Thus $E_{3,0}^{\infty}=E_{3,0}^{4}=0$ and

$$
\begin{aligned}
V\left(-4 \alpha_{-1}-3 \alpha_{0}-\alpha_{1}\right) & \cong E_{3,0}^{3} \cong E_{0,2}^{3} \cong E_{0,2}^{2} / \operatorname{Im}\left(d_{2}: E_{2,1}^{2} \rightarrow E_{0,2}^{2}\right) \\
& \cong \Lambda^{2}\left(I_{-2}\right) / \operatorname{Im}\left(d_{2}: E_{2,1}^{2} \rightarrow E_{0,2}^{2}\right)
\end{aligned}
$$

Since all the modules involved here are completely reducible over $A_{1}^{(1)}$, we have

$$
\operatorname{Im}\left(d_{2}: E_{2,1}^{2} \rightarrow E_{0,2}^{2}\right) \cong \Lambda^{2}\left(I_{-2}\right) / V\left(-4 \alpha_{-1}-3 \alpha_{0}-\alpha_{1}\right) .
$$

We have seen that the homomorphism $d_{2}: E_{4,0}^{2} \rightarrow E_{2,1}^{2}$ is trivial. Thus

$$
\begin{aligned}
E_{2,1}^{\infty} & =E_{2,1}^{3}=\operatorname{Ker}\left(d_{2}: E_{2,1}^{2} \rightarrow E_{0,2}^{2}\right) / \operatorname{Im}\left(d_{2}: E_{4,0}^{2} \rightarrow E_{2,1}^{2}\right) \\
& =\operatorname{Ker}\left(d_{2}: E_{2,1}^{2} \rightarrow E_{0,2}^{2}\right) .
\end{aligned}
$$

Since

$$
\begin{aligned}
\operatorname{Im} d_{2} & \cong \Lambda^{2}\left(I_{-2}\right) / V\left(-4 \alpha_{-1}-3 \alpha_{0}-\alpha_{1}\right) \\
& \cong E_{2,1}^{2} / \operatorname{Ker} d_{2} \cong I_{-2} \otimes I_{-2} / \operatorname{Ker} d_{2}
\end{aligned}
$$

we have

$$
\operatorname{Ker} d_{2} \cong S^{2}\left(I_{-2}\right) \oplus V\left(-4 \alpha_{-1}-3 \alpha_{0}-\alpha_{1}\right) .
$$

Therefore in either case, we have

$$
E_{3,0}^{\infty} \oplus E_{2,1}^{\infty} \cong S^{2}\left(I_{-2}\right) \oplus V\left(-4 \alpha_{-1}-3 \alpha_{0}-\alpha_{1}\right) .
$$

Now consider the sequence

$$
0 \rightarrow E_{5,0}^{2} \rightarrow E_{3,1}^{2} \rightarrow E_{1,2}^{2} \rightarrow 0 .
$$

By comparing levels, we see that the homomorphism $d_{2}: E_{3,1}^{2} \rightarrow E_{1,2}^{2}$ is trivial. Thus

$$
E_{1,2}^{3}=E_{1,2}^{2} \cong V \otimes \Lambda^{2}\left(I_{-2}\right) .
$$

Again by comparing the levels of the terms in the sequence

$$
0 \rightarrow E_{4,0}^{3} \stackrel{d_{3}}{\rightarrow} E_{1,2}^{3} \rightarrow 0,
$$

we conclude that $d_{3}=0$. Therefore $E_{1,2}^{\infty}=E_{1,2}^{4}=E_{1,2} \cong V \otimes \Lambda^{2}\left(I_{-2}\right)$.

Finally, since $E_{0,3}^{\infty}$ is a submodule of $E_{0,3}^{2} \cong \Lambda^{3}\left(I_{-2}\right)$, we see that

$$
H_{3}\left(L_{-}^{(3)}\right) \cong V\left(-4 \alpha_{-1}-3 \alpha_{0}-\alpha_{1}\right) \oplus S^{2}\left(I_{-2}\right) \oplus\left(V \otimes \Lambda^{2}\left(I_{-2}\right)\right) \oplus M,
$$

where $M$ is a direct sum of level 6 irreducible representations of $A_{1}^{(1)}$. Therefore we have

$$
H_{3}\left(L_{-}^{(3)}\right)_{-4} \cong V\left(-4 \alpha_{-1}-3 \alpha_{0}-\alpha_{1}\right) \oplus S^{2}\left(I_{-2}\right)
$$

and

$$
\begin{aligned}
I_{-4} & \cong V \otimes I_{-3} / H_{3}\left(L_{-}^{(3)}\right)_{-4} \\
& \cong V \otimes I_{-3} /\left(V\left(-4 \alpha_{-1}-3 \alpha_{0}-\alpha_{1}\right) \oplus S^{2}\left(I_{-2}\right)\right) .
\end{aligned}
$$


To determine $I_{-5}$, we study the short exact sequence

$$
0 \rightarrow N_{-}^{(3)} \rightarrow L_{-}^{(4)} \rightarrow L_{-}^{(3)} \rightarrow 0,
$$

and the corresponding spectral sequence $\left\{E_{p, q}^{r}\right\}$ converging to $H_{*}\left(L_{-}^{(4)}\right)$ such that

$$
E_{p, q}^{2} \cong H_{p}\left(L_{-}^{(3)}\right) \otimes \Lambda^{q}\left(I_{-3}\right) \text {. }
$$

We will compute $H_{3}\left(L_{-}^{(4)}\right)_{-5}$ from this spectral sequence. It is easy to show that $d_{2}: E_{2,0}^{2} \rightarrow E_{0,1}^{2}$ is an isomorphism and that $E_{2,0}^{\infty}=0$.

Consider the sequence

$$
0 \rightarrow E_{3,0}^{2} \stackrel{d_{2}}{\rightarrow} E_{1,1}^{2} \rightarrow 0 .
$$

We have

$$
E_{3,0}^{2} \cong V\left(-4 \alpha_{-1}-3 \alpha_{0}-\alpha_{1}\right) \oplus S^{2}\left(I_{-2}\right) \oplus\left(V \otimes \Lambda^{2}\left(I_{-2}\right)\right) \oplus M,
$$

and

$$
E_{1,1}^{2} \cong H_{1}\left(L_{-}^{(3)}\right) \otimes I_{-3} \cong V \otimes I_{-3} .
$$

By elementary linear algebra, we have $E_{3,0}^{2} \cong \operatorname{Ker} d_{2} \oplus \operatorname{Im} d_{2}$. Since $d_{2}$ is a homomorphism between $A_{1}^{(1)}$-modules, by comparing levels, we see that $d_{2}$ maps $\left(V \otimes \Lambda^{2}\left(I_{-2}\right)\right) \oplus M$ to zero. Thus Im $d_{2}$ is isomorphic to a submodule of $V\left(-4 \alpha_{-1}-3 \alpha_{0}-\alpha_{1}\right) \oplus S^{2}\left(I_{-2}\right)$. By Lemma 3.2 and (6.5), we have

$$
H_{2}\left(L_{-}^{(4)}\right) \cong I_{-4} \cong V \otimes I_{-3} /\left(V\left(-4 \alpha_{-1}-3 \alpha_{0}-\alpha_{1}\right) \oplus S^{2}\left(I_{-2}\right)\right) .
$$

Since

$$
E_{1,1}^{\infty}=E_{1,1}^{3}=E_{1,1}^{2} / \operatorname{Im} d_{2} \cong V \otimes I_{-3} / \operatorname{Im} d_{2}
$$

is a direct summand of $H_{2}\left(L_{-}^{(4)}\right), \operatorname{Im} d_{2}$ contains a submodule isomorphic to $V\left(-4 \alpha_{-1}-3 \alpha_{0}-\alpha_{1}\right) \oplus S^{2}\left(I_{-2}\right)$. Hence we conclude that

$$
\operatorname{Im} d_{2} \cong V\left(-4 \alpha_{-1}-3 \alpha_{0}-\alpha_{1}\right) \oplus S^{2}\left(I_{-2}\right) \text {. }
$$

Therefore

$$
E_{3,0}^{3} \cong\left(V \otimes \Lambda^{2}\left(I_{-2}\right)\right) \oplus M
$$

and $E_{1,1}^{\infty}=E_{1,1}^{3} \cong I_{-4}$. This implies that $E_{0,2}^{\infty}=E_{0,2}^{4}=0$. Thus the homomorphism $d_{3}$ is surjective in the sequence

$$
0 \rightarrow E_{3,0}^{3} \stackrel{d_{3}}{\rightarrow} E_{0,2}^{3} \rightarrow 0 .
$$

Since $E_{0,2}^{3}$ is a submodule of $E_{0,2}^{2}=\Lambda^{2}\left(I_{-3}\right)$, by comparing levels, we see that $\operatorname{Ker} d_{3}$ must contain $V \otimes \Lambda^{2}\left(I_{-2}\right)$. It follows that

$$
E_{3,0}^{\infty}=E_{3,0}^{4} \cong\left(V \otimes \Lambda^{2}\left(I_{-2}\right)\right) \oplus M^{\prime},
$$

where $M^{\prime}$ is a direct sum of level 6 irreducible highest weight representations of $A_{1}^{(1)}$. Therefore $\left(E_{3,0}^{\infty}\right)_{-5} \cong V \otimes \Lambda^{2}\left(I_{-2}\right)$. It is easy to see that

$$
\left(E_{2,1}^{\infty}\right)_{-5}=\left(E_{1,2}^{\infty}\right)_{-5}=\left(E_{0,3}^{\infty}\right)_{-5}=0 .
$$

Therefore we have

$$
H_{3}\left(L_{-}^{(4)}\right)_{-5} \cong V \otimes \Lambda^{2}\left(I_{-2}\right)
$$


and

$$
I_{-5} \cong V \otimes I_{-4} / H_{3}\left(L_{-}^{(4)}\right)_{-5} \cong V \otimes I_{-4} / V \otimes \Lambda^{2}\left(I_{-2}\right) .
$$

Case 2. $H A_{2}^{(2)}$.

For the structure of $I_{-4}$, we study the spectral sequence $\left\{E_{p, q}^{r}\right\}$ corresponding to the short exact sequence

$$
0 \rightarrow N_{-}^{(2)} \rightarrow L_{-}^{(3)} \rightarrow L_{-}^{(2)} \rightarrow 0
$$

As in the case of $H A_{1}^{(1)}$, it is easy to deduce that the map $d_{2}: E_{2,0}^{2} \rightarrow E_{0,1}^{2}$ is an isomorphism and that $E_{2,0}^{\infty}=0$.

By comparing the levels of the terms in the sequence

$$
0 \rightarrow E_{3,0}^{2} \stackrel{d_{2}}{\rightarrow} E_{1,1}^{2} \rightarrow 0
$$

we get $d_{2}=0$. So

$$
E_{3,0}^{3}=E_{3,0}^{2}=V\left(-6 \alpha_{-1}-5 \alpha_{0}-\alpha_{1}\right),
$$

and

$$
E_{1,1}^{\infty}=E_{1,1}^{3}=E_{1,1}^{2} \cong V \otimes I_{-2}=I_{-3} .
$$

Since $H_{2}\left(L_{-}^{(3)}\right) \cong I_{-3}$, we have $E_{0,2}^{\infty}=E_{0,2}^{4}=0$. Hence the homomorphism $d_{3}: E_{3,0}^{3} \rightarrow E_{0,2}^{3}$ is surjective. But since $E_{0,2}^{\infty}$ is a submodule of $E_{0,2}^{2} \cong$ $\Lambda^{2}\left(I_{-2}\right)$, we see that $d_{3}$ is trivial. This implies

$$
E_{3,0}^{\infty}=E_{3,0}^{4}=E_{3,0}^{3}=V\left(-6 \alpha_{-1}-5 \alpha_{0}-\alpha_{1}\right)
$$

and $E_{0,2}^{3}=0$. Thus the homomorphism $d_{2}: E_{2,1}^{2} \rightarrow E_{0,2}^{2}$ is surjective in the following sequence

$$
0 \rightarrow E_{4,0}^{2} \rightarrow E_{2,1}^{2} \rightarrow E_{0,2}^{2} \rightarrow 0 \text {. }
$$

Again by comparing levels, we deduce that the homomorphism $d_{2}: E_{4,0}^{2} \rightarrow E_{2,1}^{2}$ must be trivial. Therefore

$$
E_{4,0}^{3}=E_{4,0}^{2}=V\left(-9 \alpha_{-1}-8 \alpha_{0}-2 \alpha_{1}\right)
$$

and

$$
\begin{aligned}
E_{2,1}^{\infty} & =E_{2,1}^{3}=\operatorname{Ker}\left(d_{2}: E_{2,1}^{2} \rightarrow E_{0,2}^{2}\right) / \operatorname{Im}\left(d_{2}: E_{4,0}^{2} \rightarrow E_{2,1}^{2}\right) \\
& \cong \operatorname{Ker}\left(d_{2}: E_{2,1}^{2} \rightarrow E_{0,2}^{2}\right)
\end{aligned}
$$

Since

$$
\operatorname{Im} d_{2} \cong \Lambda^{2}\left(I_{-2}\right) \cong E_{2,1}^{2} / \operatorname{Ker} d_{2} \cong I_{-2} \otimes I_{-2} / \operatorname{Ker} d_{2},
$$

we have $\operatorname{Ker} d_{2} \cong S^{2}\left(I_{-2}\right)$. Therefore $E_{2,1}^{\infty} \cong S^{2}\left(I_{-2}\right)$.

Now consider the sequence

$$
0 \rightarrow E_{5,0}^{2} \rightarrow E_{3,1}^{2} \rightarrow E_{1,2}^{2} \rightarrow 0 \text {. }
$$

By comparing levels, we see that the homomorphism $d_{2}: E_{3,1}^{2} \rightarrow E_{1,2}^{2}$ is trivial. Thus $E_{1,2}^{3}=E_{1,2}^{2} \cong V \otimes \Lambda^{2}\left(I_{-2}\right)$. Again by comparing the levels of the terms in the sequence

$$
0 \rightarrow E_{4,0}^{3} \stackrel{d_{3}}{\rightarrow} E_{1,2}^{3} \rightarrow 0
$$


we conclude $d_{3}=0$. Therefore

$$
E_{1,2}^{\infty}=E_{1,2}^{4}=E_{1,2}^{3} \cong V \otimes \Lambda^{2}\left(I_{-2}\right) .
$$

Finally, since $E_{0,3}^{\infty}$ is a submodule of $E_{0,3}^{2} \cong \Lambda^{3}\left(I_{-2}\right)$, we see that

$$
H_{3}\left(L_{-}^{(3)}\right) \cong V\left(-6 \alpha_{-1}-5 \alpha_{0}-\alpha_{1}\right) \oplus S^{2}\left(I_{-2}\right) \oplus\left(V \otimes \Lambda^{2}\left(I_{-2}\right)\right) \oplus M,
$$

where $M$ is a direct sum of level 6 irreducible highest weight representations of $A_{2}^{(2)}$. Therefore we obtain

$$
H_{3}\left(L_{-}^{(3)}\right)_{-4} \cong S^{2}\left(I_{-2}\right)
$$

and

$$
I_{-4} \cong V \otimes I_{-3} / H_{3}\left(L_{-}^{(3)}\right)_{-4} \cong V \otimes I_{-3} / S^{2}\left(I_{-2}\right) .
$$

To determine $I_{-5}$, we study the spectral sequence $\left\{E_{p, q}^{r}\right\}$ corresponding to the short exact sequence

$$
0 \rightarrow N_{-}^{(3)} \rightarrow L_{-}^{(4)} \rightarrow L_{-}^{(3)} \rightarrow 0 .
$$

As in the case of $H A_{1}^{(1)}$, we know that

$$
\left(E_{3,0}^{\infty}\right)_{-5} \cong V \otimes \Lambda^{2}\left(I_{-2}\right)
$$

and that

$$
\left(E_{2,1}^{\infty}\right)_{-5}=\left(E_{1,2}^{\infty}\right)_{-5}=\left(E_{0,3}^{\infty}\right)_{-5}=0
$$

Therefore we have

$$
H_{3}\left(L_{-}^{(4)}\right)_{-5} \cong V \otimes \Lambda^{2}\left(I_{-2}\right)
$$

and

$$
I_{-5} \cong V \otimes I_{-4} / H_{3}\left(L_{-}^{(4)}\right)_{-5} \cong V \otimes I_{-4} / V \otimes \Lambda^{2}\left(I_{-2}\right) .
$$

Case 3. $H A_{l}^{(1)}, l \geq 2, H C_{2}^{(1)}, H D_{4}^{(3)}$.

We first study the case of $H A_{l}^{(1)}$. Consider the short exact sequence

$$
0 \rightarrow N_{-}^{(2)} \rightarrow L_{-}^{(3)} \rightarrow L_{-}^{(2)} \rightarrow 0,
$$

and the corresponding spectral sequence $\left\{E_{p, q}^{r}\right\}$. As we have seen before, $d_{2}: E_{2,0}^{2} \rightarrow E_{0,1}^{2}$ is an isomorphism and hence $E_{2,0}^{\infty}=0$.

In the sequence

$$
0 \rightarrow E_{3,0}^{2} \stackrel{d_{2}}{\rightarrow} E_{1,1}^{2} \rightarrow 0
$$

we have

$$
E_{3,0}^{2} \cong H_{3}\left(L_{-}^{(2)}\right) \cong V\left(-3 \alpha_{-1}-2 \alpha_{0}-\alpha_{1}\right) \oplus V\left(-3 \alpha_{-1}-2 \alpha_{0}-\alpha_{l}\right),
$$

and $E_{1,1}^{2} \cong V \otimes I_{-2}$. As we have seen before, we have

$$
\operatorname{Im} d_{2} \cong V\left(-3 \alpha_{-1}-2 \alpha_{0}-\alpha_{1}\right) \oplus V\left(-3 \alpha_{-1}-2 \alpha_{0}-\alpha_{l}\right) .
$$

Hence $E_{3,0}^{3}=0$, which implies $E_{3,0}^{\infty}=0$.

Moreover, since $E_{1,1}^{\infty}=E_{1,1}^{3} \cong I_{-3}$, we have $E_{0,2}^{\infty}=E_{0,2}^{4}=0$. Thus the homomorphism $d_{3}$ is surjective in the sequence

$$
0 \rightarrow E_{3,0}^{3} \stackrel{d_{3}}{\rightarrow} E_{0,2}^{3} \rightarrow 0
$$


Since $E_{3,0}^{3}=0$, we also have $E_{0,2}^{3}=0$. That is, the homomorphism $d_{2}: E_{2,1}^{2}$ $\rightarrow E_{0,2}^{2}$ is surjective in the sequence

$$
0 \rightarrow E_{4,0}^{2} \rightarrow E_{2,1}^{2} \rightarrow E_{0,2}^{2} \rightarrow 0 .
$$

Note that

$$
E_{4,0}^{2} \cong V\left(-4 \alpha_{-1}-3 \alpha_{0}-2 \alpha_{1}-\alpha_{2}\right) \oplus V\left(-4 \alpha_{-1}-3 \alpha_{0}-2 \alpha_{1}-\alpha_{l-1}\right)
$$

and that

$$
E_{2,1}^{2} \cong I_{-2} \otimes I_{-2} \cong V\left(-2 \alpha_{-1}-\alpha_{0}\right) \otimes V\left(-2 \alpha_{-1}-\alpha_{0}\right) .
$$

In the decomposition of $V\left(-2 \alpha_{-1}-\alpha_{0}\right) \otimes V\left(-2 \alpha_{-1}-\alpha_{0}\right)$, every irreducible component must have the form $V(\lambda-m \delta)$, with $m \geq 0$ and

$$
-4 \alpha_{-1}-2 \alpha_{0}-\delta<\lambda \leq-4 \alpha_{-1}-2 \alpha_{0} .
$$

Since $\delta=\alpha_{0}+\alpha_{1}+\cdots+\alpha_{l}, \lambda$ must satisfy

$$
-4 \alpha_{-1}-2 \alpha_{0}-\lambda<\alpha_{0}+\alpha_{1}+\cdots+\alpha_{l} .
$$

But we have

$$
-4 \alpha_{-1}-2 \alpha_{0}-\left(-4 \alpha_{-1}-3 \alpha_{0}-2 \alpha_{1}-\alpha_{2}\right)=\alpha_{0}+2 \alpha_{1}+\alpha_{2} \nless \delta,
$$

and

$$
-4 \alpha_{-1}-2 \alpha_{0}-\left(-4 \alpha_{-1}-3 \alpha_{0}-2 \alpha_{1}-\alpha_{l-1}\right)=\alpha_{0}+2 \alpha_{1}+\alpha_{l-1} \nless \delta .
$$

Thus $V\left(-4 \alpha_{-1}-3 \alpha_{0}-2 \alpha_{1}-\alpha_{2}\right)$ and $V\left(-4 \alpha_{-1}-3 \alpha_{0}-2 \alpha_{1}-\alpha_{l-1}\right)$ do not occur as irreducible components in the decomposition of

$$
V\left(-2 \alpha_{-1}-\alpha_{0}\right) \otimes V\left(-2 \alpha_{-1}-\alpha_{0}\right) .
$$

Therefore the homomorphism $d_{2}: E_{4,0}^{2} \rightarrow E_{2,1}^{2}$ is trivial and we obtain

$$
E_{2,1}^{\infty}=E_{2,1}^{3} \cong I_{-2} \otimes I_{-2} / \operatorname{Im}\left(d_{2}: E_{2,1}^{2} \rightarrow E_{0,2}^{2}\right) \cong S^{2}\left(I_{-2}\right) .
$$

It is easy to show that $\left(E_{1,2}^{\infty}\right)_{-4}=\left(E_{0,3}^{\infty}\right)_{-4}=0$. Hence we get

$$
H_{3}\left(L_{-}^{(3)}\right)_{-4} \cong S^{2}\left(I_{-2}\right) \text {, }
$$

and

$$
I_{-4} \cong V \otimes I_{-3} / S^{2}\left(I_{-2}\right) .
$$

The proofs for the other cases are similar.

Case 4. $H A_{2 l}^{(2)}, H D_{l+1}^{(2)}, l \geq 2$.

By the same argument of Case 1, we have

$$
H_{3}\left(L_{-}^{(3)}\right)_{-4} \cong S^{2}\left(I_{-2}\right) \oplus V\left(-4 \alpha_{-1}-3 \alpha_{0}-\alpha_{1}\right),
$$

and therefore

$$
I_{-4} \cong V \otimes I_{-3} /\left(V\left(-4 \alpha_{-1}-3 \alpha_{0}-\alpha_{1}\right) \oplus S^{2}\left(I_{-2}\right)\right) .
$$




\section{THE PRINCIPALLY SPECIALIZED AFFINE CHARACTERS AND THE ROOT MULTIPLICITIES}

In this section, we compute the principally specialized affine characters and the root multiplicities of the Lorentzian Kac-Moody Lie algebras $\mathfrak{g}(\widetilde{A})$ for certain higher levels. We define the signum function as follows. If $\alpha$ is an integer, then

$$
\begin{aligned}
\varepsilon(\alpha, t) & =1 & & \text { if } \alpha \text { is divisible by } t, \\
& =0 & & \text { otherwise, }
\end{aligned}
$$

and if $\alpha$ is a root of a Kac-Moody Lie algebra $\mathfrak{g}(A)$, then

$$
\begin{array}{rlrl}
\varepsilon(\alpha, t)=1 & & \text { if } \alpha \text { is divisible by a root of level } t, \\
& =0 & & \text { otherwise. }
\end{array}
$$

Let $F(q)=\sum_{n=0}^{\infty} f(n) q^{n}, X(q), Y(q)$, and $Z(q)$ be the principally specialized characters of the integrable irreducible representations of the affine KacMoody Lie algebras $\mathfrak{g}(A)$ of level 1, 2, 3, and 4 given in [Kang].

In $\S 2$, we have seen that the Witt formula gives the principally specialized affine characters of the homogeneous subspaces of the maximal graded Lie algebra $G$ :

$$
\begin{aligned}
\operatorname{ch}_{q} G_{-l} & =\sum_{m \geq 0}\left(\operatorname{dim} G_{(l, m)}\right) q^{m} \\
& =\sum_{m \geq 0}\left(\sum_{(r, s) \mid(l, m+l)} \mu\left(\frac{(l, m+l)}{(r, s)}\right) \frac{(r, s)}{(l, m+l)} \widehat{B}(r, s)\right) q^{m},
\end{aligned}
$$

where the function $\widehat{B}(r, s)$ is defined by the formula (2.7). We introduce the following functions:

$$
\begin{aligned}
W_{2}(q) & =\operatorname{ch}_{q} G_{-2} \\
& =\sum_{m \geq 0}\left(\widehat{B}(2, m+2)-\frac{1}{2} \varepsilon(m, 2) f\left(\frac{m}{2}\right)\right) q^{m}, \\
W_{3}(q) & =\operatorname{ch}_{q} G_{-3} \\
& =\sum_{m \geq 0}\left(\widehat{B}(3, m+3)-\frac{1}{3} \varepsilon(m, 3) f\left(\frac{m}{3}\right)\right) q^{m}, \\
W_{4}(q)= & \operatorname{ch}_{q} G_{-4} \\
= & \sum_{m \geq 0}\left(\widehat{B}(4, m+4)-\frac{1}{2} \varepsilon(m, 2) \widehat{B}\left(2, \frac{m}{2}+2\right)\right) q^{m}, \\
W_{5}(q) & =\operatorname{ch}_{q} G_{-5} \\
& =\sum_{m \geq 0}\left(\widehat{B}(5, m+5)-\frac{1}{5} \varepsilon(m, 5) f\left(\frac{m}{5}\right)\right) q^{m} .
\end{aligned}
$$

Theorem 7.1. Let $L=\bigoplus_{n \in Z} L_{n}$ be the realization of the Lorentzian Kac-Moody Lie algebra $\mathfrak{g}(\widetilde{A})$. Then the principally specialized affine characters of $L_{-n}$, $n=1,2, \ldots, 5$, are given by the following formulas. 
$H A_{1}^{(1)}: \quad \operatorname{ch}_{q} L_{-1}=F(q)$,

$$
\begin{aligned}
& \operatorname{ch}_{q} L_{-2}=W_{2}(q)-X(q), \\
& \operatorname{ch}_{q} L_{-3}=W_{3}(q)-F(q) X(q), \\
& \operatorname{ch}_{q} L_{-4}=W_{4}(q)-F(q)^{2} X(q)+Z(q)+\frac{1}{2}\left(X(q)^{2}+X\left(q^{2}\right)\right), \\
& \operatorname{ch}_{q} L_{-5}=W_{5}(q)-F(q)^{3} X(q)+F(q) Z(q)+F(q) X(q)^{2} .
\end{aligned}
$$

$H A_{2}^{(2)}: \quad \operatorname{ch}_{q} L_{-1}=F(q)$,

$$
\begin{aligned}
& \operatorname{ch}_{q} L_{-2}=W_{2}(q)-X(q) \\
& \operatorname{ch}_{q} L_{-3}=W_{3}(q)-F(q) X(q) \\
& \operatorname{ch}_{q} L_{-4}=W_{4}(q)-F(q)^{2} X(q)+\frac{1}{2}\left(X(q)^{2}+X\left(q^{2}\right)\right), \\
& \operatorname{ch}_{q} L_{-5}=W_{5}(q)-F(q)^{3} X(q)+F(q) X(q)^{2} .
\end{aligned}
$$

$H A_{l}^{(1)}, l \geq 2$ :

$$
\begin{aligned}
& \operatorname{ch}_{q} L_{-1}=F(q), \\
& \operatorname{ch}_{q} L_{-2}=W_{2}(q)-X(q), \\
& \operatorname{ch}_{q} L_{-3}=W_{3}(q)-F(q) X(q)+2 Y(q), \\
& \operatorname{ch}_{q} L_{-4}=W_{4}(q)-F(q)^{2} X(q)+2 F(q) Y(q)+\frac{1}{2}\left(X(q)^{2}+X\left(q^{2}\right)\right) .
\end{aligned}
$$

$H C_{2}^{(1)}, H D_{4}^{(3)}$ :

$$
\begin{aligned}
& \operatorname{ch}_{q} L_{1}=F(q) \\
& \operatorname{ch}_{q} L_{-2}=W_{2}(q)-X(q) \\
& \operatorname{ch}_{q} L_{-3}=W_{3}(q)-F(q) X(q)+Y(q) \\
& \operatorname{ch}_{q} L_{-4}=W_{4}(q)-F(q)^{2} X(q)+F(q) Y(q)+\frac{1}{2}\left(X(q)^{2}+X\left(q^{2}\right)\right) .
\end{aligned}
$$

$H A_{2 l}^{(2)}, H D_{l+1}^{(2)}, l \geq 2$ :

$\operatorname{ch}_{q} L_{-1}=F(q)$,

$\operatorname{ch}_{q} L_{-2}=W_{2}(q)-X(q)$,

$\operatorname{ch}_{q} L_{-3}=W_{3}(q)-F(q) X(q)$,

$\operatorname{ch}_{q} L_{-4}=W_{4}(q)-F(q)^{2} X(q)+Z(q)+\frac{1}{2}\left(X(q)^{2}+X\left(q^{2}\right)\right)$.

$H A_{2 l-1}^{(2)}, H B_{l}^{(1)}, H C_{l}^{(1)}, l \geq 3, H D_{l}^{(1)}, l \geq 4, H F_{4}^{(1)}, H G_{2}^{(1)}, H E_{6}^{(1)}$, $H E_{7}^{(1)}, H E_{8}^{(1)}, H E_{6}^{(2)}: \quad \operatorname{ch}_{q} L_{-1}=F(q)$,

$$
\begin{aligned}
& \operatorname{ch}_{q} L_{-2}=W_{2}(q)-X(q), \\
& \operatorname{ch}_{q} L_{-3}=W_{3}(q)-F(q) X(q)+Y(q) .
\end{aligned}
$$

Proof. The results follow directly from Theorem 6.2 and formula (2.8).

Now we compute the root multiplicities. We first consider the hyperbolic Kac-Moody Lie algebra $H A_{1}^{(1)}$. Let $V$ be the basic representation of $A_{1}^{(1)}$ and 
let $S=\left\{\tau_{i} \mid i=1,2,3, \ldots\right\}$ be an enumeration of all the weights of $V$. Define the function $B^{(1)}(\tau)$ by

$$
B^{(1)}(\tau)=\sum_{(n) \in T(\tau)} \frac{\left(\left(\sum n_{i}\right)-1\right) !}{\prod\left(n_{i} !\right)} \prod\left(p\left(1-\frac{\left(\tau_{i} \mid \tau_{i}\right)}{2}\right)\right)^{n_{i}},
$$

where $(\mid)$ denotes the standard invariant symmetric bilinear form on $\mathfrak{h}^{*}$ [Kac2]. Then by the Witt formula, we have

$$
\operatorname{dim}\left(G_{-}\right)_{\alpha}=\sum_{\tau \mid \alpha} \mu\left(\frac{\alpha}{\tau}\right) \frac{\tau}{\alpha} B^{(1)}(\tau) .
$$

Thus for the roots of level 2, the Witt formula gives

$$
\operatorname{dim}\left(G_{-2}\right)_{\alpha}=B^{(1)}(\alpha)-\frac{1}{2} \varepsilon(\alpha, 2) p\left(1-\frac{1}{8}(\alpha \mid \alpha)\right) .
$$

Since $I_{-2} \cong V\left(-2 \alpha_{-1}-\alpha_{0}\right)$, by [F-L], we have

$$
\operatorname{dim}\left(I_{-2}\right)_{\alpha}=E\left(3-\frac{(\alpha \mid \alpha)}{2}\right),
$$

where the coefficients $E(n)$ are given by the equation

$$
\sum_{n=0}^{\infty} E(n) q^{n}=\frac{1}{\prod_{n \geq 1}\left(1-q^{4 n}\right)\left(1-q^{4 n-1}\right)\left(1-q^{4 n-3}\right)}=\frac{\phi\left(q^{2}\right)}{\phi(q) \phi\left(q^{4}\right)} .
$$

Therefore we obtain

$$
\operatorname{dim}\left(L_{-2}\right)_{\alpha}=B^{(1)}(\alpha)-\frac{1}{2} \varepsilon(\alpha, 2) p\left(1-\frac{1}{8}(\alpha \mid \alpha)\right)-E\left(3-\frac{(\alpha \mid \alpha)}{2}\right) .
$$

For the roots of level 3, by the Witt formula, we have

$$
\operatorname{dim}\left(G_{-3}\right)_{\alpha}=B^{(1)}(\alpha)-\frac{1}{3} \varepsilon(\alpha, 3) p\left(1-\frac{1}{18}(\alpha \mid \alpha)\right) .
$$

By Theorem 5.2,

$$
I_{-3} \cong V \otimes I_{-2} \cong V\left(-\alpha_{-1}\right) \otimes V\left(-2 \alpha_{-1}-\alpha_{0}\right) .
$$

In [Fe1], Feingold showed that $I_{-3}$ has a decomposition into a direct sum of level 3 irreducible representations of the affine Kac-Moody Lie algebra $A_{1}^{(1)}$ :

$$
I_{-3}=\sum_{m \geq 0}\left(a_{m} V\left(-3 \alpha_{-1}-\alpha_{0}-m \delta\right)+b_{m} V\left(-3 \alpha_{-1}-(m+1) \delta\right)\right),
$$

where the coefficients $a_{m}$ and $b_{m}$ are given by

$$
a_{m}=\sum_{j \in \mathbf{Z}}(p(m-j(20 j+3))-p(m-(4 j+3)(5 j+3)))
$$

and

$$
b_{m}=\sum_{j \in \mathbf{Z}}\left(p\left(m-\left(20 j^{2}+11 j+1\right)\right)-p\left(m-\left(20 j^{2}+19 j+4\right)\right)\right) .
$$

Therefore, by $[\mathrm{F}-\mathrm{L}]$, we have

$$
\begin{aligned}
N^{(1)}(\alpha) & =\operatorname{dim}\left(I_{-3}\right)_{\alpha} \\
& =\sum_{m \geq 0}\left(a_{m} H\left(1-3 m-\frac{(\alpha \mid \alpha)}{2}\right)+b_{m} G\left(6-3 m-\frac{(\alpha \mid \alpha)}{2}\right)\right),
\end{aligned}
$$


where the functions $G$ and $H$ are defined recursively as follows. For $m>0$,

$$
\begin{gathered}
0=\sum_{n \in \mathbf{Z}}(-1)^{n} G\left(3 m-\frac{1}{2} n(5 n+3)\right), \\
0=\sum_{n \in \mathbf{Z}}(-1)^{n} G\left(3 m-1-\frac{1}{2} n(5 n+1)\right),
\end{gathered}
$$

with the initial conditions $G(0)=1, G(-3 m)=G(-3 m+2)=0$, and for $m>0$,

$$
\begin{gathered}
0=\sum_{n \in \mathbf{Z}}(-1)^{n} H\left(3 m-\frac{1}{2} n(5 n+3)\right), \\
0=\sum_{n \in \mathbf{Z}}(-1)^{n} H\left(3 m+2-\frac{1}{2} n(5 n+1)\right),
\end{gathered}
$$

with the initial conditions $H(2)=1, H(-3 m+3)=H(-3 m+2)=0$. Therefore we have

$$
\operatorname{dim}\left(L_{-3}\right)_{\alpha}=B^{(1)}(\alpha)-\frac{1}{3} \varepsilon(\alpha, 3) p\left(1-\frac{1}{18}(\alpha \mid \alpha)\right)-N^{(1)}(\alpha) .
$$

We summarize these results in the following theorem.

Theorem 7.2. Let $L=\bigoplus_{n \in Z} L_{n}$ be the realization of the hyperbolic Kac-Moody Lie algebra $H A_{1}^{(1)}$. Then we have the following root multiplicity formulas:

$$
\begin{aligned}
& \operatorname{dim}\left(L_{-1}\right)_{\alpha}=p\left(1-\frac{(\alpha \mid \alpha)}{2}\right), \\
& \operatorname{dim}\left(L_{-2}\right)_{\alpha}=B^{(1)}(\alpha)-\frac{1}{2} \varepsilon(\alpha, 2) p\left(1-\frac{1}{8}(\alpha \mid \alpha)\right)-E\left(3-\frac{(\alpha \mid \alpha)}{2}\right), \\
& \operatorname{dim}\left(L_{-3}\right)_{\alpha}=B^{(1)}(\alpha)-\frac{1}{3} \varepsilon(\alpha, 3) p\left(1-\frac{1}{18}(\alpha \mid \alpha)\right)-N^{(1)}(\alpha) .
\end{aligned}
$$

For the hyperbolic Kac-Moody Lie algebra $H A_{2}^{(2)}$, let $S=\left\{\tau_{i} \mid i=1,2,3, \ldots\right\}$ be an enumeration of all the weights of the basic representation $V$ of $A_{2}^{(2)}$. Define the function $B^{(2)}(\tau)$ by

$$
B^{(2)}(\tau)=\sum_{(n) \in T(\tau)} \frac{\sum\left(\left(n_{i}\right)-1\right) !}{\prod\left(n_{i} !\right)} \prod\left(p\left(\frac{1}{2}-\frac{\left(\tau_{i} \mid \tau_{i}\right)}{2}\right)\right)^{n_{i}} .
$$

Then by the Witt formula, we have

$$
\operatorname{dim}\left(G_{-}\right)_{\alpha}=\sum_{\tau \mid \alpha} \mu\left(\frac{\alpha}{\tau}\right) \frac{\tau}{\alpha} B^{(2)}(\tau) .
$$

Thus for the roots of level 2, the Witt formula gives

$$
\operatorname{dim}\left(G_{-2}\right)_{\alpha}=B^{(2)}(\alpha)-\frac{1}{2} \varepsilon(\alpha, 2) p\left(\frac{1}{2}-\frac{1}{8}(\alpha \mid \alpha)\right) .
$$

Since $I_{-2} \cong V\left(-2 \alpha_{-1}-\alpha_{0}\right)$, by [F-L], we have

$$
\operatorname{dim}\left(I_{-2}\right)_{\alpha}=D(4-(\alpha \mid \alpha)) \text {, }
$$

where the function $D$ is defined recursively as follows. For $k>0$,

$$
\sum_{n \in \mathbf{Z}} D(4 k-n(15 n+4))=\sum_{n \in \mathbf{Z}} D(4 k-(3 n+1)(5 n+3)),
$$


and for $m>1$,

$$
\sum_{n \in \mathbf{Z}} D(4 m-3-n(15 n+2))=\sum_{n \in \mathbf{Z}} D(4 m-3-(3 n+1)(5 n+1)),
$$

with the initial conditions $D(1)=1, D(-4 m+8)=D(-4 m+5)=0$. Therefore we obtain

$$
\operatorname{dim}\left(L_{-2}\right)_{\alpha}=B^{(2)}(\alpha)-\frac{1}{2} \varepsilon(\alpha, 2) p\left(\frac{1}{2}-\frac{1}{8}(\alpha \mid \alpha)\right)-D(4-(\alpha \mid \alpha)) .
$$

For the roots of level 3 , by the Witt formula, we have

$$
\operatorname{dim}\left(G_{-3}\right)_{\alpha}=B^{(2)}(\alpha)-\frac{1}{3} \varepsilon(\alpha, 3) p\left(\frac{1}{2}-\frac{1}{18}(\alpha \mid \alpha)\right) .
$$

By Theorem 6.2,

$$
I_{-3} \cong V \otimes I_{-2} \cong V\left(-\alpha_{-1}\right) \otimes V\left(-2 \alpha_{-1}-\alpha_{0}\right)
$$

and $I_{-3}$ has a decomposition into a direct sum of level 3 irreducible representations of the affine Kac-Moody Lie algebra $A_{2}^{(2)}$ [Fel]:

$$
I_{-3}=\sum_{m \geq 0}\left(a_{m} V\left(-3 \alpha_{-1}-\alpha_{0}-m \delta\right)+b_{m} V\left(-3 \alpha_{-1}-(m+1) \delta\right)\right),
$$

where the coefficients $a_{m}$ and $b_{m}$ are given by

$$
a_{m}+\sum_{j \in \mathbf{Z}}(p(m-j(15 j-2))-p(m-(5 j+1)(3 j+1))),
$$

and

$$
b_{m}=\sum_{j \in \mathbf{Z}}(p(m-j(15 j-7))-p(m-(5 j+1)(3 j+2))) .
$$

Therefore by $[\mathrm{F}-\mathrm{L}]$ we have

$$
\begin{aligned}
N^{(2)}(\alpha) & =\operatorname{dim}\left(I_{-3}\right)_{\alpha} \\
& =\sum_{m \geq 0}\left(a_{m} K\left(\frac{9}{2}-3 m-\frac{(\alpha \mid \alpha)}{2}\right)+b_{m} J\left(\frac{3}{2}-3 m-\frac{(\alpha \mid \alpha)}{2}\right)\right),
\end{aligned}
$$

where the functions $J$ and $K$ are defined recursively as follows. For $k>0$,

$$
\sum_{n \in \mathbf{Z}} J(3 k-3 n(3 n+1))=\sum_{n \in \mathbf{Z}} J(3 k-(3 n+1)(3 n+2)),
$$

and for $m>0$,

$$
\sum_{n \in \mathbf{Z}} J\left(3 m-2-(3 n)^{2}\right)=\sum_{n \in \mathbf{Z}} J\left(3 m-2-(3 n+1)^{2}\right),
$$

with the initial conditions $J(0)=1, J(-3 m)=J(-3 m+1)=0$. For $k>0$,

$$
\sum_{n \in \mathbf{Z}} K(3 k-3 n(3 n+1))=\sum_{n \in \mathbf{Z}} K(3 k-(3 n+1)(3 n+2)),
$$

and for $m>1$,

$$
\sum_{n \in \mathbf{Z}} K\left(3 m-2-(3 n)^{2}\right)=\sum_{n \in \mathbf{Z}} K\left(3 m-2-(3 n+1)^{2}\right),
$$

with the initial conditions $K(1)=1, K(-3 m+6)=K(-3 m+4)=0$. Therefore we have

$$
\operatorname{dim}\left(L_{-3}\right)_{\alpha}=B^{(2)}(\alpha)-\frac{1}{3} \varepsilon(\alpha, 3) p\left(\frac{1}{2}-\frac{1}{18}(\alpha \mid \alpha)\right)-N^{(2)}(\alpha) .
$$


We summarize the above results in the following theorem.

Theorem 7.3. Let $L=\bigoplus_{n \in \mathrm{Z}} L_{n}$ be the realization of the hyperbolic Kac-Moody Lie algebra $\mathrm{HA}_{2}^{(2)}$. Then we have the following root multiplicity formulas:

$$
\begin{aligned}
& \operatorname{dim}\left(L_{-1}\right)_{\alpha}=p\left(\frac{1}{2}-\frac{(\alpha \mid \alpha)}{2}\right), \\
& \operatorname{dim}\left(L_{-2}\right)_{\alpha}=B^{(2)}(\alpha)-\frac{1}{2} \varepsilon(\alpha, 2) p\left(\frac{1}{2}-\frac{1}{8}(\alpha \mid \alpha)\right)-D(4-(\alpha \mid \alpha)), \\
& \operatorname{dim}\left(L_{-3}\right)_{\alpha}=B^{(2)}(\alpha)-\frac{1}{3} \varepsilon(\alpha, 3) p\left(\frac{1}{2}-\frac{1}{18}(\alpha \mid \alpha)\right)-N^{(2)}(\alpha) .
\end{aligned}
$$

Remark 7.4. Recently, Kac has informed the author that he also discovered a level 3 root multiplicity formula for the hyperbolic Kac-Moody Lie algebra $H A_{1}^{(1)}$. We introduce his formula in the following. Unfortunately, the proof has not been available to the author yet.

Define a function $\omega(k)$ by

$$
\begin{aligned}
\omega(k) & =1 & & \text { if } k \equiv 0,1(\bmod 3), \\
& =0 & & \text { if } k \equiv-1(\bmod 3) .
\end{aligned}
$$

Then we have

$$
\operatorname{dim}\left(L_{-3}\right)_{\alpha}=\nu\left(1-\frac{(\alpha \mid \alpha)}{2}\right),
$$

where the coefficients $\nu(n)$ are defined by

$$
\begin{aligned}
\sum_{n \geq 0} \nu(n) q^{n+8}= & \frac{\phi\left(q^{24}\right)}{3 \phi\left(q^{3}\right)^{2} \phi\left(q^{6}\right)}\left(2 \frac{\phi\left(q^{24}\right)}{\phi\left(q^{6}\right)} \prod_{j \equiv \pm 3(\bmod 24)}\left(1+q^{j}\right)-3\right) \\
& \times\left(q^{2} \prod_{j \equiv \pm 9(\bmod 24)}\left(1+q^{j}\right) \sum_{k \in \mathbf{Z}} \omega(k) q^{2 k^{2}+2 k}\right. \\
& \left.+q^{3} \prod_{j \equiv \pm 3(\bmod 24)}\left(1+q^{j}\right) \sum_{k \in \mathbf{Z}} \omega(k) q^{2 k^{2}}\right) \\
& +\frac{1}{3}\left(\frac{1}{\phi\left(q^{3}\right) \phi\left(q^{6}\right)} \sum_{k \in \mathbf{Z}} \omega(k) q^{2 k^{2}}-\frac{1}{\phi\left(q^{9}\right)}\right) .
\end{aligned}
$$

Combining (7.23) with (7.13) yields the following combinatorial identity:

$$
B^{(1)}(\alpha)-\frac{1}{3} \varepsilon(\alpha, 3) p\left(1-\frac{1}{18}(\alpha \mid \alpha)\right)-N^{(1)}(\alpha)=\nu\left(1-\frac{(\alpha \mid \alpha)}{2}\right) .
$$

\section{ACKNOWLEDGMENTS}

This paper is part of my thesis at Yale University. I would like to express my sincere gratitude to Professor George B. Seligman for his invaluable guidance and encouragement throughout my graduate study at Yale University. 


\section{REFERENCES}

[B-K-M] B. Benkart, S.-J. Kang, and K. Misra, Graded Lie algebras of Kac-Moody type, Adv. in Math. 97 (1993), 154-190.

[B-M] S. Berman and R. V. Moody, Multiplicities in Lie algebras, Proc. Amer. Math. Soc. 76 (1979), 223-228.

[B] N. Bourbaki, Groupes et algèbres de Lie, Hermann, Paris, 1968, Chapitres 4-6.

[C-E] H. Cartan and S. Eilenberg, Homological algebra, Princeton Univ. Press, Princeton, N.J., 1956.

[Fe1] A. J. Feingold, Tensor products of certain modules for the generalized Cartan matrix Lie algebra $A_{1}^{(1)}$, Comm. Algebra 9 (1981), 1323-1341.

[Fe2] - Some applications of vertex operators to Kac-Moody algebras, Vertex Operators in Mathematics and Physics, Springer-Verlag, New York, 1984, pp. 185-206.

[F-F] A. J. Feingold and I. B. Frenkel, $A$ hyperbolic Kac-Moody algebra and the theory of Siegel modular forms of genus 2, Math. Ann. 263 (1983), 87-144.

[F-L] A. J. Feingold and J. Lepowsky, The Weyl-Kac character formula and power series identities, Adv. Math. 29 (1978), 271-309.

[Fr] I. B. Frenkel, Representations of Kac-Moody algebras and dual resonance models, Applications of Group Theory in Physics and Mathematical Physics, Lectures in Appl. Math., vol. 21, Amer. Math. Soc., Providence, R.I., 1985, pp. 325-353.

[F-K] I. B. Frenkel and V. G. Kac, Basic representations of affine Lie algebras and dual resonance models, Invent. Math. 62 (1980), 23-66.

[F] D. B. Fuks, Cohomology of infinite-dimensional Lie algebras, Consultants Bureau, Plenum, New York, 1986.

[G-K] O. Gabber and V. G. Kac, On defining relations of certain infinite dimensional Lie algebras, Bull. Amer. Math. Soc. 5 (1981), 185-189.

[G-L] H. Garland and J. Lepowsky, Lie algebra homology and the MacDonald-Kac formulas, Invent. Math. 34 (1976), 37-76.

[H-S] G. Hochschild and J. P. Serre, Cohomology of Lie algebras, Ann. of Math. (2) 57 (1953), 591-603.

[J1] N. Jacobson, Basic algebra. II, Freeman, San Francisco, Calif., 1980.

[J2] _ Lie algebras, 2nd ed., Dover, New York, 1979.

[Kac1] V. G. Kac, Simple irreducible graded Lie algebras of finite growth, Math. USSR-Izv. 2 (1968), 1271-1311.

[Kac2] _ Infinite dimensional Lie algebras, 2nd ed., Cambridge Univ. Press, Cambridge and New York, 1985.

[K-M-W] V. G. Kac, R. V. Moody, and M. Wakimoto, On $E_{10}$, differential geometrical methods in theoretical physics (K. Bleuler and M. Werner, eds.), Kluwer Academic Publishers, 1988, pp. 109-128.

[Kang] S.-J. Kang, Gradations and structure of Kac-Moody Lie algebras, Dissertation, Yale Univ., 1990.

[Ko] B. Kostant, Lie algebra cohomology and the generalized Borel-Weil theorem, Ann. of Math. (2) 74 (1961), 329-387.

[L] J. Lepowsky, Application of the numerator formula to $k$-rowed plane partitions, Adv. Math. 35 (1980), 175-194.

[L-M] J. Lepowsky and R. V. Moody, Hyperbolic Lie algebras and quasi-regular cusps on Hilbert modular surfaces, Math. Ann. 245 (1979), 63-88.

[Liu] L.-S. Liu, Kostant's formula in Kac-Moody Lie algebras, Dissertation, Yale Univ., 1990.

[Mcd] I. G. Macdonald, Affine root systems and Dedekind's $\eta$-function, Invent. Math. 15 (1972), 91-143. 
[Ma] O. Mathieu, Formules de caractères pour les algèbres de Kac-Moody générales, Astérisque 159-160 (1988).

[Mc1] J. McCleary, User's guide to spectral sequences, Math. Lectures Ser. 12, Publish or Perish, 1985.

[M1] R. V. Moody, A new class of Lie algebras, J. Algebra 10 (1968), 211-230.

[M2] _ Root systems of hyperbolic type, Adv. Math. 33 (1979), 144-160.

[Mo-T] R. E. Mosher and M. C. Tangora, Cohomology operations and applications in homotopy theory, Harper and Row, New York, 1968.

[Se] J. P. Serre, Lie algebras and Lie groups, 1964 Lectures given at Harvard University, Benjamin, New York, 1965.

Department of Mathematics, Yale University, New Haven, Connecticut 06520

Current address: Department of Mathematics, University of Notre Dame, Notre Dame, Indiana 46556 